\title{
Insights into the regulation of intrinsically disordered proteins in the human proteome by analyzing sequence and gene expression data Yvonne JK Edwards ${ }^{\star}$, Anna E Lobley ${ }^{\star}$, Melissa M Pentony and David T Jones
}

Address: Bioinformatics Group, Department of Computer Science, University College London, Gower Street, London, WC1E 6BT, UK.

$\bowtie$ These authors contributed equally to this work.

Correspondence: David T Jones. Email: d.jones@cs.ucl.ac.uk

Published: II May 2009

Genome Biology 2009, 10:R50 (doi:10.I I86/gb-2009-10-5-r50)

The electronic version of this article is the complete one and can be found online at http://genomebiology.com/2009/10/5/R50
Received: 16 December 2008

Revised: 23 March 2009

Accepted: II May 2009

(C) 2009 Edwards et al.; licensee BioMed Central Ltd.

This is an open access article distributed under the terms of the Creative Commons Attribution License (http://creativecommons.org/licenses/by/2.0), which permits unrestricted use, distribution, and reproduction in any medium, provided the original work is properly cited.

\begin{abstract}
Background: Disordered proteins need to be expressed to carry out specified functions; however, their accumulation in the cell can potentially cause major problems through protein misfolding and aggregation. Gene expression levels, mRNA decay rates, microRNA (miRNA) targeting and ubiquitination have critical roles in the degradation and disposal of human proteins and transcripts. Here, we describe a study examining these features to gain insights into the regulation of disordered proteins.

Results: In comparison with ordered proteins, disordered proteins have a greater proportion of predicted ubiquitination sites. The transcripts encoding disordered proteins also have higher proportions of predicted miRNA target sites and higher mRNA decay rates, both of which are indicative of the observed lower gene expression levels. The results suggest that the disordered proteins and their transcripts are present in the cell at low levels and/or for a short time before being targeted for disposal. Surprisingly, we find that for a significant proportion of highly disordered proteins, all four of these trends are reversed. Predicted estimates for miRNA targets, ubiquitination and mRNA decay rate are low in the highly disordered proteins that are constitutively and/or highly expressed.

Conclusions: Mechanisms are in place to protect the cell from these potentially dangerous proteins. The evidence suggests that the enrichment of signals for miRNA targeting and ubiquitination may help prevent the accumulation of disordered proteins in the cell. Our data also provide evidence for a mechanism by which a significant proportion of highly disordered proteins (with high expression levels) can escape rapid degradation to allow them to successfully carry out their function.
\end{abstract}




\section{Background}

Natively unfolded or disordered proteins are proteins that do not form a stable three-dimensional structure in their native state. A disordered protein can be either completely unfolded or comprise both folded and unfolded segments [1-4]. Previous analyses have shown that the presence of large regions of disorder within proteins correlates strongly with function [120]. These functions typically relate to gene regulation and signaling classes that are of particular importance to higher organisms $[6,21]$. Previous work has also shown that over $30 \%$ of proteins in eukaryotic genomes are likely to be disordered, a percentage that is much higher than found within prokaryotic genomes [6,12,22,23]. Whilst there are functional benefits that accrue from disordered proteins, the use of disorder carries with it significant risks [24]. The prevalence of human diseases that correspond to highly disordered proteins is striking [24-31]; these include diabetes, neurodegenerative disorders [25-28], cardiovascular disease [29] and cancer [30]. In fact, many neurodegenerative disorders arise from the aggregation of disordered proteins [25-28]. If disordered proteins are indeed potential hazards to the healthy maintenance of human cells, then both their production and disposal should be very carefully regulated. Such is the danger of protein aggregation in living cells that a number of efficient degradation mechanisms are in place to quickly dispose of misfolded proteins [32]. The problem for disordered proteins may well be to survive long enough to carry out their function in such a hostile environment.

The equilibrium level of a protein depends on its rate of production relative to its rate of degradation. The quantity of a protein produced in the cell is affected by the expression level of its mRNA transcript. The levels of gene expression are controlled in the cell in a number of different ways - for example, by varying the rates of transcription and translation and altering the rate at which mRNA is degraded. In combination with transcription, mRNA degradation plays a critical role in regulating gene expression [33,34]. If proteins need to remain in the disordered state for any length of time, they need to either bypass the endogenous degradation pathways (such as the ATP-dependent proteolytic $26 \mathrm{~S}$ proteasome [32]) that specifically target unfolded proteins or be produced in sufficient quantity to temporarily overload the protein degradation pathways. The second option is, of course, extremely risky as high production levels of disordered proteins may result in aggregation. This suggests that the first option is the most likely, but in this case, how can disordered proteins escape rapid degradation to allow them to successfully carry out their function.

Recent work suggested that disordered residues make a protein more susceptible to intracellular degradation [35]. The in vivo half-lives of yeast proteins were shown to correlate with disorder as opposed to the actual degradation signals and motifs. In our study we analyze biological properties known to regulate and affect the degradation rates of proteins and transcripts to investigate how these correlate with protein disorder. Gene expression is a continuous process spanning transcription factor activation, nuclear localization of transcription factors, chromatin decompaction, coupled initiation and 5 ' capping of transcripts, coupled transcription and mRNA processing, splicing, cleavage and 3' polyadenylation, mRNA packaging, mRNA export into the cytoplasm, translation and protein folding [36]. Biological processes that lower the mRNA copy numbers include proteolytic degradation by proteases, microRNA (miRNA):mRNA targeting and destruction of mRNA by nucleases. Here, we characterize absolute mRNA levels, mRNA decay rates, protein stability, predicted miRNA targeting and ubiquitination to assess whether disordered proteins (and their encoding transcripts) display any unusual characteristics.

miRNAs are a class of small non-coding RNA molecules (comprising about 22 nucleotides) that regulate gene expression and mediate diverse cellular processes such as development, differentiation, proliferation and apoptosis [37-41]. miRNAs target the 3 ' untranslated regions of mRNA molecules, which typically results in the down-regulation of gene expression by translational repression and/or a reduction of mRNA transcript levels [42]. Several algorithms are available to predict the mRNA targets [43-51].

Ubiquitination is a reversible post-translational modification of cellular proteins where ubiquitin (a 76 residue protein) is covalently attached to the $\varepsilon$ amino group of lysines of target proteins. Diverse forms of ubiquitin modifications exist and influence the functional outcome of target proteins in distinct ways [52,53]. Mono-ubiquitination or multi-ubiquitination are implicated in various nonproteolytic cellular functions, including endocytosis, endosomal sorting and DNA repair [52]. Polyubiquitination is mainly associated with proteasomal degradation $[54,55]$. Whilst ubiquitination can determine the fate of a given protein for proteolytic degradation by the $26 \mathrm{~S}$ proteosome, ubiquitination of transcription factors with a VP-16 activation domain is also shown to be required for transcriptional activation [56-58]. Like miRNA targeting [59-69], ubiquitination is crucial in regulating a variety of cellular processes in eukaryotes [59-61] and has significant implications in the etiology of a number of serious diseases such as cancer [62-64], neurodegeneration [65,66] and cardiovascular dysfunction [67-69].

To gain new insights into the regulation of disordered proteins, we carried out a series of studies to examine how a number of features known to affect protein and transcript degradation correlate with protein disorder. We investigated whether the mRNA transcripts encoding disordered proteins decay more rapidly. To establish mRNA expression patterns for transcripts encoding disordered proteins and to reveal novel insights into the molecular mechanisms of transcriptional regulation [70-74], mRNA expression levels were characterized in normal tissues and cell lines using public domain 
microarray expression datasets. Transcripts co-expressed with the transcripts encoding disordered proteins were identified to suggest the key biological pathways that are affected or under regulatory control of disordered proteins and their transcripts. We investigated whether disordered proteins have lower expression levels and/or the transcripts encoding them are more likely to be targeted by miRNA. One of the aims of this analysis was to use miRNA prediction to establish the trends that exist between possible miRNA targeting and the transcripts encoding disordered proteins. We examined if disordered proteins contain sites that are more susceptible to degradation using a novel ubiquitination site prediction tool. Protein turnover rates for disordered sequences were also investigated by considering stability determined from an in vivo study measuring protein turnover [75].

In this study, we examine the available human gene expression data and properties of the human proteome and transcriptome to investigate whether disordered proteins have any unusual characteristics in terms of their production and disposal in human cells. Specifically, we were interested in gaining insights into the means by which disordered proteins avoid early degradation without resorting to the severe risks of over-expression.

\section{Results}

Five properties of the human proteins and transcripts were investigated in relation to disorder in the proteome. First, three expression profile studies on transcripts encoding disordered proteins were carried out: the general features of their expression levels were characterized; their expression profiles across the samples were clustered by abundance and functionally annotated to provide a classification of the biological roles of their encoded proteins; and transcripts coexpressed with them were identified. Second, we searched for correlation between the extent of mRNA decay rates and varying amounts of protein disorder encoded by transcripts. Third, the occurrence of disorder was compared with protein stability indices determined by a global stability profiling assay. Fourth, miRNA prediction tools were used to establish trends that exist between transcripts encoding disordered proteins and miRNA targeting. Finally, correlations between ubiquitination sites and protein disorder levels were investigated.

\section{Protein disorder and gene expression}

Protein disorder and absolute gene expression levels

On average, transcripts that encode highly disordered proteins are expressed in lower copy numbers than those that encode highly ordered proteins (Figure 1a). Figure 1a shows the average absolute gene expression values calculated across 207 normal tissue and cell line samples (Table 1). Whilst the scale for the absolute values is displayed in $\log _{2}$ units, in the decimal scale the absolute gene expression levels of the genes for transcripts that encode highly disordered proteins are roughly half those of the genes for transcripts that encode highly ordered proteins. A similar trend was obtained for transcripts that encode disordered and ordered proteins (Figure S1a in Additional data file 1).

To investigate whether these low expression levels were correlated with occurrence of disorder in the protein products, transcripts were grouped according to the frequency of disorder in the encoded protein (Figure 2a). As the percentage of disordered residues increases to between $>60 \%$ and $\leq 80 \%$ (or from now on $(60,80] \%$ in standard interval notation), the average gene expression level steadily decreases. However, for the $(80,100] \%$ disorder category the average sample expression levels were greater than expected using a Wilcoxon paired rank test $(P<0.0001)$. This $(80,100] \%$ category comprises $<1 \%$ of the data (Table 2 ). To verify that these trends were independent of function, we filtered the data to impose equality of representation of biological process (BP) and molecular function (MF) Gene Ontology (GO) terms. Specifically, a maximum of ten randomly chosen examples were selected for each annotation term at specificity level 4 or

Table I

\begin{tabular}{|c|c|c|c|c|}
\hline Dataset & Description & Samples & Cel file sample replicates & References \\
\hline [GEO:GSEII33] & Normal tissues and cell lines & 144 & $72 \times 2$ & [7I] \\
\hline [GEO:GSE236I] & Normal human tissues & 36 & $36 \times 1$ & {$[72]$} \\
\hline \multirow[t]{4}{*}{ [GEO:GSE2004] } & Normal spleen & 22 & $3 \times 3$ (spleen) & - \\
\hline & liver and kidney & & $2 \times 3$ (liver) & \\
\hline & & & I × 3 (liver) & \\
\hline & & & I × 4 (kidney) & \\
\hline [GEO:GSE78I] & Normal kidney samples & 5 & $1 \times 5$ & [70] \\
\hline Total & & 207 & 75 & \\
\hline
\end{tabular}




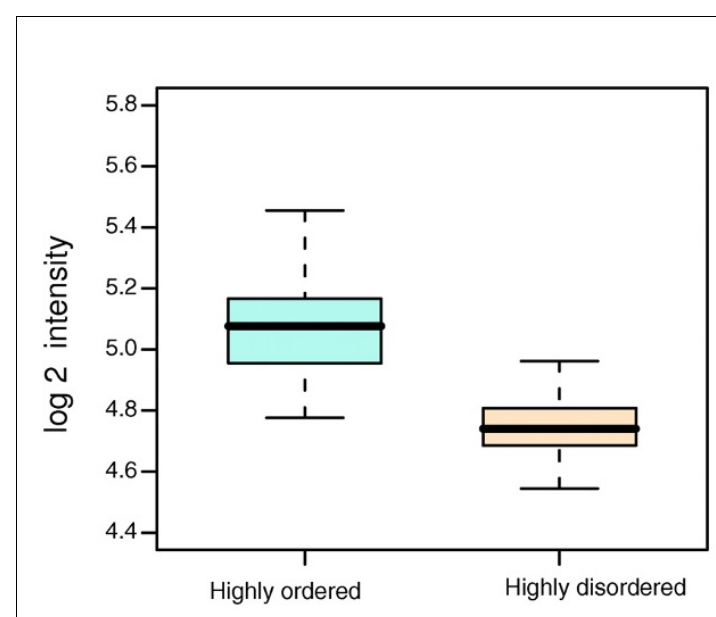

(a) Microarray expressions

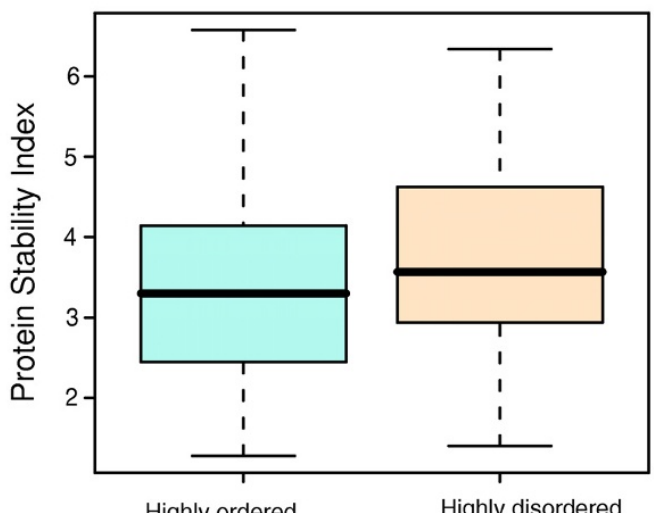

(c) Protein stability index

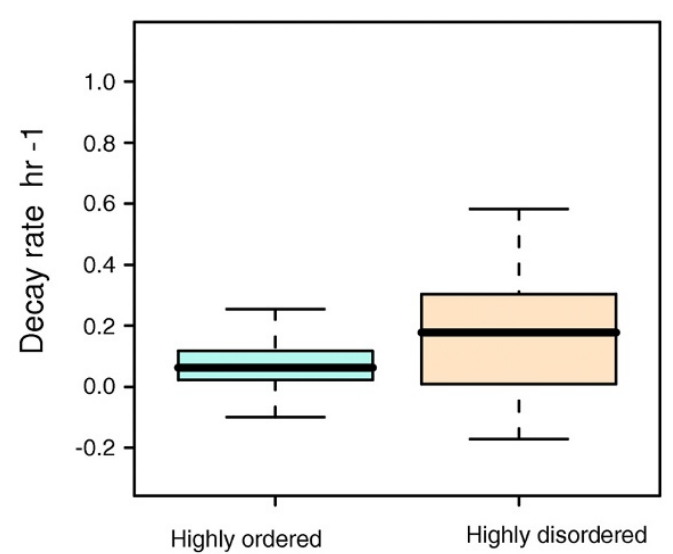

(b) mRNA decay rates

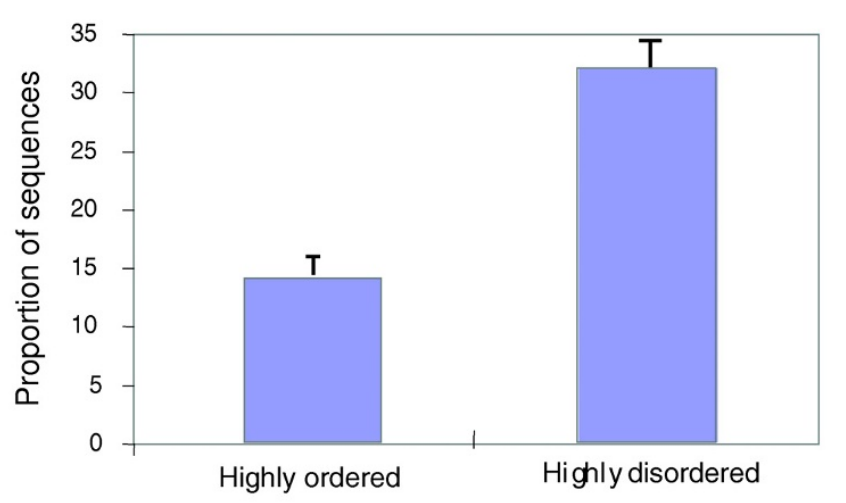

(d) miRNA targetting

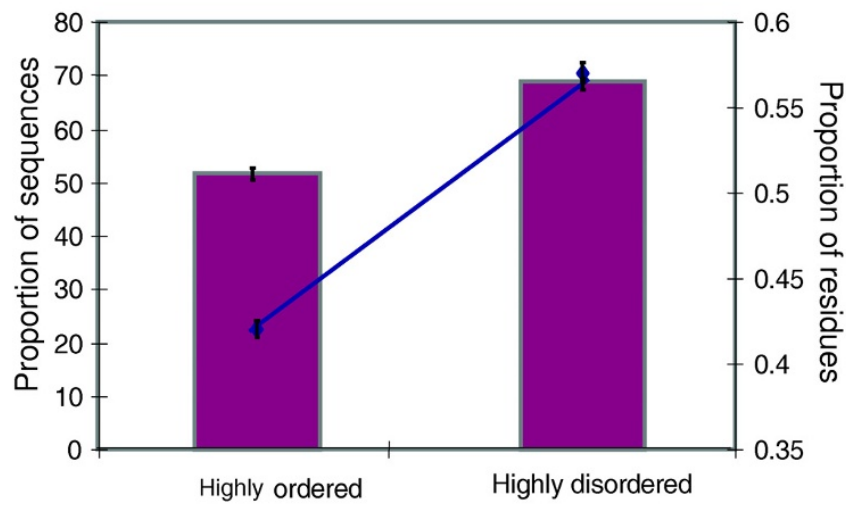

(e) Ubiquitin targetting

\section{Figure I}

Properties of highly ordered and highly disordered proteins. (a) Box-plot distributions of the average expression levels for the transcripts encoding the highly ordered and the highly disordered proteins. (b) Box-plot of mRNA decay rates for the highly ordered and highly disordered proteins. (c) Box-plot of protein stability values. (d) The percentage of transcripts likely to be regulated by miRNA ( $y$-axis) for the transcripts encoding the highly ordered and the highly disordered proteins. (e) The percentage of the proteins with one or more predicted ubiquitination sites (principal y-axis, burgundy bar chart) in the highly ordered and the highly disordered datasets; and the percentage of residues predicted as ubiquitination sites (secondary $y$-axis, navy line plot) versus different amounts of disorder. 
Table 2

\begin{tabular}{|c|c|c|c|c|}
\hline & Total* & Uniquet & Match $\ddagger$ & Percentage§ \\
\hline \multicolumn{5}{|l|}{ Category of disorder } \\
\hline Highly disordered & 877 & 827 & 257 & 31.08 \\
\hline Highly ordered & 5,693 & 5,351 & 782 & $|4.6|$ \\
\hline Disordered & 15,095 & 14,282 & 5,056 & 35.40 \\
\hline Ordered & 18,774 & 17,766 & 3,433 & 19.32 \\
\hline All proteins & 33,869 & 32,010 & 8,468 & 26.45 \\
\hline \multicolumn{5}{|c|}{ Percentage of disorder } \\
\hline \multicolumn{5}{|l|}{ Disordered } \\
\hline$[0,20]$ & 4,271 & 4,055 & $\mathrm{I}, 402$ & 34.57 \\
\hline$(20,40]$ & 6,957 & 6,603 & 2,300 & 34.83 \\
\hline$(40,60]$ & 3,036 & 2,866 & 1,119 & 39.04 \\
\hline$(60,80]$ & 679 & 644 & 233 & 36.18 \\
\hline$(80,100]$ & 152 & 143 & 20 & 13.99 \\
\hline Total & 15,095 & $|4,3| \mid$ & 5,074 & 35.45 \\
\hline \multicolumn{5}{|l|}{ Ordered } \\
\hline$[0,20]$ & $|6,34|$ & 15,503 & 3,037 & 19.59 \\
\hline$(20,40]$ & 2,173 & 2,024 & 362 & 17.89 \\
\hline$(40,60]$ & 214 & 207 & 35 & 16.91 \\
\hline$(60,80]$ & 33 & 31 & 4 & 12.9 \\
\hline$(80,100]$ & 13 & 9 & 0 & 0 \\
\hline Total & 18,774 & 17,774 & 3,438 & 19.34 \\
\hline \multicolumn{5}{|l|}{ Proteome } \\
\hline$[0,20]$ & 20,612 & 19,536 & 4,429 & 22.67 \\
\hline$(20,40]$ & 9,130 & 8,618 & 2,658 & 30.84 \\
\hline$(40,60]$ & 3,250 & 3,073 & $\mathrm{I}, \mathrm{I} 54$ & 37.55 \\
\hline$(60,80]$ & 712 & 675 & 237 & 35.11 \\
\hline$(80,100]$ & 165 & 152 & 20 & 13.16 \\
\hline Total & 33,869 & 32,010 & 8,468 & 26.45 \\
\hline
\end{tabular}

For each data set, the *total number of transcripts encoding proteins and the tnumber of unique protein sequences encoded by transcripts are given. $¥ A$ match occurs when a transcript of a protein sequence matches an mRNA targeted by a miRNA. \$The percentage calculations are described in the Materials and methods. Values according to the category of disorder (Figures Ic, 2c) and the percentages of disordered residues (Figure $3 \mathrm{c}$ ) are given.

below. The results (Figure 2a) indicate that the correlation between transcript expression levels and the amount of disorder are not dictated by function class bias and represent genuine and robust features of the data.

Absolute gene expression profiles for highly disordered proteins To differentiate modes of gene expression behavior among the highly disordered proteins, hierarchical clustering analysis of the absolute expression levels was carried out. The resulting heat map (Figure 3a) shows that the situation is not as simple as suggested in Figure 1. Five broad classes of expression patterns for the genes encoding highly disordered proteins could be defined (Figure 3; Tables S1 and S2 in Additional data file 2). These groups were functionally characterized by performing over-representation tests within each of the five classes. The first set of transcripts (light blue) encode proteins that are almost entirely disordered and contained within the $(80,100] \%$ disorder category. In this constitutively expressed group, all transcripts represent large ribosomal subunits that are essential parts of the transcription machinery and expressed in every cell. The second group (dark blue) represents transcripts that exhibit high expression levels in the majority of tissues and display little or no tissue specificity. The third group (green) contains transcripts expressed at medium levels. General DNA binding and transcription factor functions were over-represented in the proteins encoded by the medium expressor group. The fourth group (gold) contains transcripts expressed in a tissue-specific manner. The remaining transcripts form a group not detected to be abundant in any of the tissues studied and is referred to as the low or transient expressor group (gray). This low or transient expressor group comprises over 50\% of transcripts analyzed (Table 3) and is primarily responsible for the low expression trend reported above. This suggests that over half of the transcripts encoding proteins with large regions of disorder are expressed either at transient or low levels.

\section{Co-regulated transcripts and the highly disordered proteins}

A similar functional analysis was carried out for all transcripts detected to be significantly co-regulated with transcripts encoding disordered proteins. Co-regulation was established using significance of the correlation coefficient between transcripts and was calculated for transcript pairs in the $(60,80] \%$ and $(80,100] \%$ disorder groups. Using empirically derived $P$-values from the distribution of correlations, a significance threshold at either tail of $P<0.01$ was used to describe transcripts as co-regulated. Several of the categories identified as enriched in the co-regulated transcript datasets overlapped and are summarized. In general, the activities of the ubiquitin degradation pathway and the proteolytic catabolic processes were observed to be anti-correlated (downregulated) with the expression profiles of transcripts encoding highly disordered proteins. Functions enriched in the significantly correlated transcript set included protein complex formation, protein dimerization, protein homo-dimerization, protein hetero-oligomerization and enzyme inhibitors that reduce the activity of proteases (that is, enzymes catalyzing the hydrolysis of peptide bonds) (Table 4).

\section{Protein disorder, mRNA decay rates and protein stability indices}

The mRNA decay rates of the transcripts of 74 highly disordered proteins and 536 highly ordered proteins were compared. The mRNA decay rates for the transcripts encoding highly disordered proteins $\left(0.190871 \mathrm{~h}^{-1}\right)$ are more than twice 


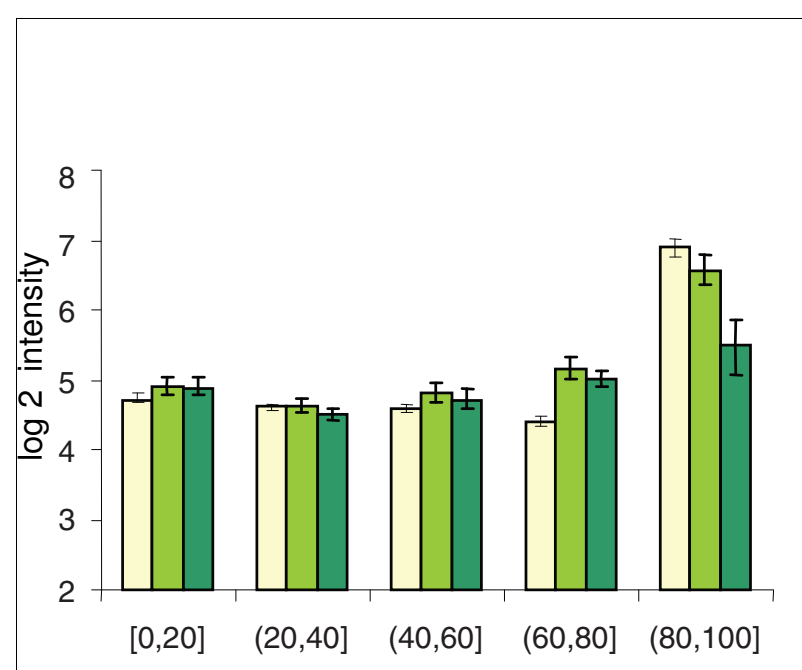

(a) Gene expression intensities

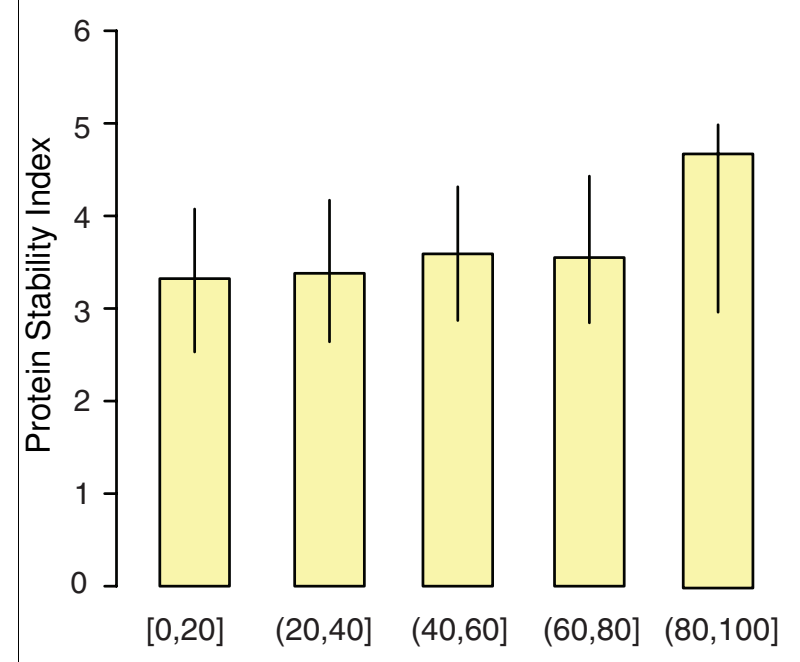

(c) Protein stability index
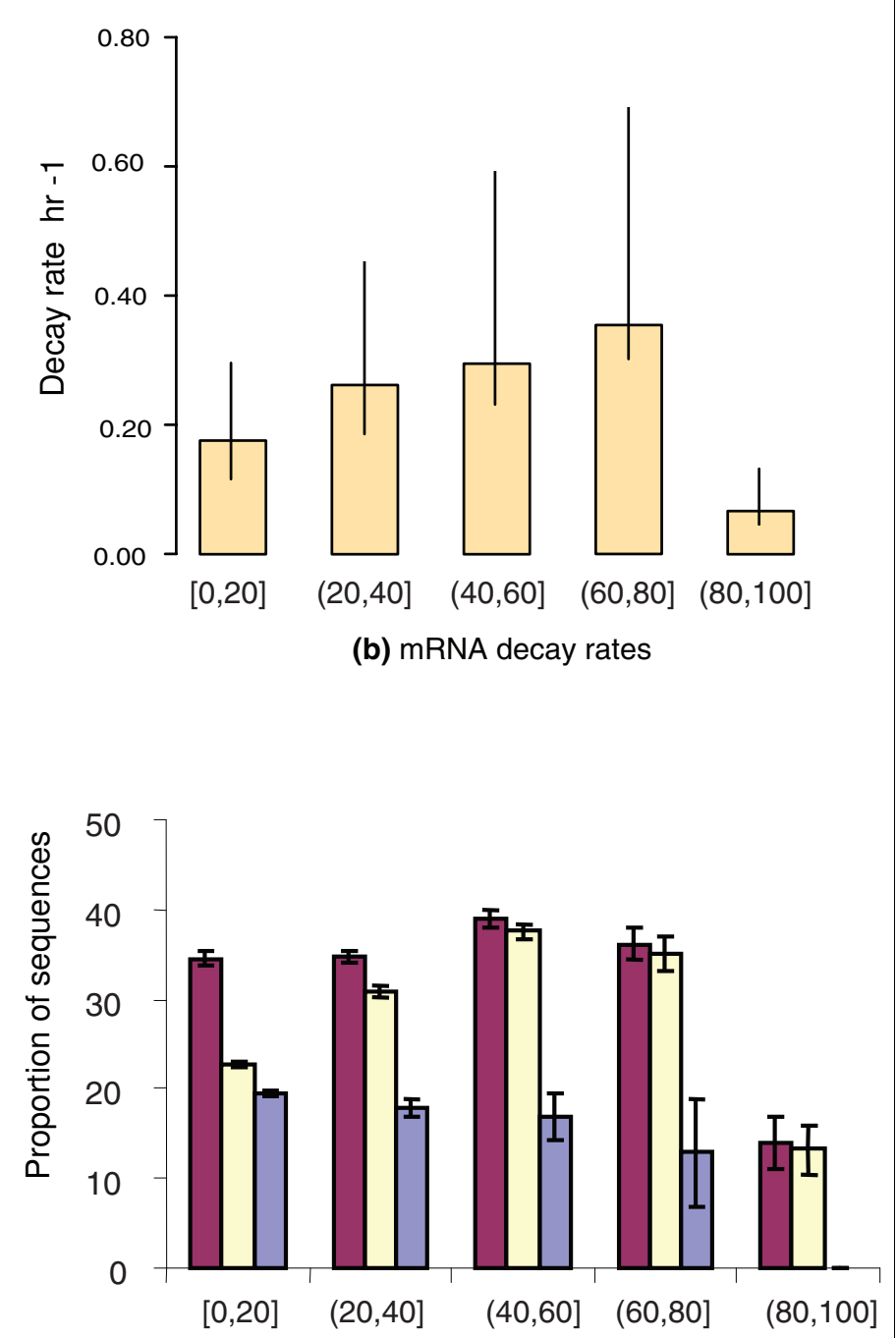

(d) miRNA targetting

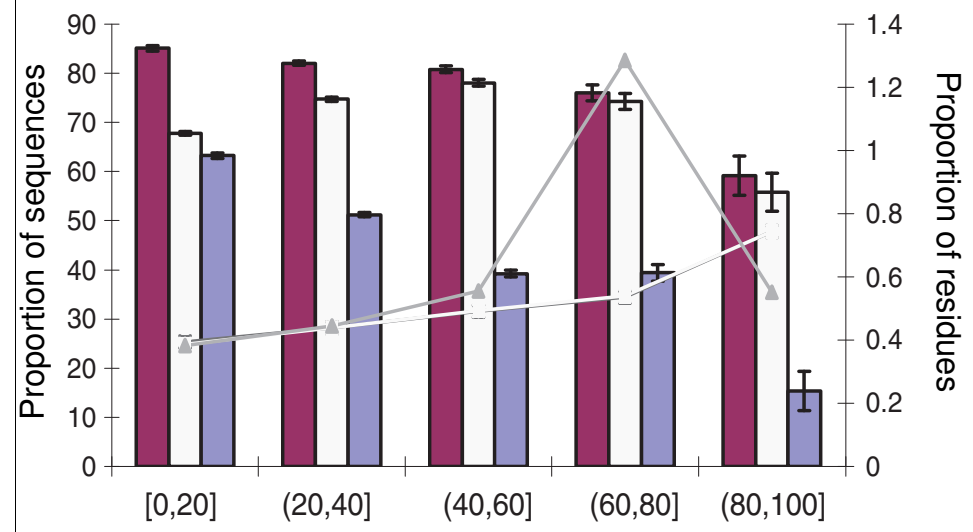

(e) Ubiquitin targetting

Figure 2 (see legend on next page) 
Figure 2 (see previous page)

Correlation of features with percentage of disorder in the proteome. (a) Variation in absolute transcript expression as the percentage of disorder increases in the proteome (yellow bars). The bar charts represent the average sample expression for the groups of transcripts separated according to the percentage range (x-axis) of the total disordered residues in the encoded proteins. The $y$-axis scale represents log2 absolute expression. Expression levels for the transcripts with MF and BP GO terms at level 4 are shown as light green and dark green bars, respectively. (b) Variation of mRNA decay rate as disorder increases in the proteome. mRNA decay rates versus the percentage bins of disordered residues are shown. (c) Variation of protein stability as disorder increases in the proteome. The stability index versus the percentage bins of disordered residues are shown. (d) The proportion of protein coding transcripts targeted by miRNA ( $y$-axis) as the percentage of disorder increases in the proteome. The datasets for the transcripts encoding the disordered proteins (burgundy) and ordered proteins (mauve) and the proteome (yellow) are shown. (e) The percentage of the proteins with one or more predicted ubiquitination sites against the percentage of disorder (principal y-axis, bar charts); and the percentage of residues predicted as ubiquitination sites against the percentage of disorder (secondary $y$-axis, line plots). The transcripts encoding the disordered proteins, the ordered proteins and the proteome are shown in burgundy, mauve and yellow (respectively).

that observed for the transcripts encoding highly ordered proteins (0.084944 $\mathrm{h}^{-1}$ ) (Figure $\left.1 \mathrm{~b}\right)$. A statistically significant difference $(P<0.02)$ between mRNA decay rates for transcripts encoding highly ordered and highly disordered proteins was found, with the highly disordered datasets having higher mRNA decay rates. The mRNA decay rates for the transcripts encoding 1,980 disordered proteins (o.177596 $\left.\mathrm{h}^{-1}\right)$ and 1,858 ordered proteins (0.096878 $\mathrm{h}^{-1}$ ) were also compared and a similar trend was obtained (Figure S1b in Additional data file 1$)$.

We divided the 33,869 proteins into bins by percentage of disordered residues. When we compared the mRNA decay rates for each of the bins (Figure 2b), there was no significant difference between them. Although this result does not suggest that all disordered proteins show a significant association with higher mRNA decay rates, it does concur with our previous analysis of the (highly) ordered and (highly) disordered protein datasets, in showing a distinct difference between mRNA decay rates for both groups.

The protein stability measures of the highly disordered (179) and highly ordered groups $(1,396)$ were also compared. We found a significant difference $(P<0.0005)$ between the halflives of highly ordered and highly disordered proteins, with highly disordered proteins having longer half-lives (Figure 1c).
Consistent with our analysis of decay rates, we divided the 8,666 disordered proteins into bins by percentage of disordered residues. Protein stability indices showed no significant affiliation to a particular binned group, although the $(80,100] \%$ disorder bin showed much higher half-lives than the other binned groups (Figure 2c).

Since trends were observed between both mRNA decay rate and disorder, and protein half-life and disorder, the half-lives and decay rates were also compared to see if a relationship existed between mRNA decay rate and protein half-life. The Pearson correlation value between 1,446 overlapping sequences (-0.06) was not significant and suggested that these two characteristics are independent.

\section{Protein disorder and miRNA targets}

Approximately one-quarter of protein coding transcripts are predicted miRNA targets (Table 2). The proportion of transcripts encoding highly disordered proteins that are likely to be miRNA targets is approximately twice that of transcripts encoding highly ordered proteins (Figure 1d; Table 2). The frequency of transcripts with at least one predicted miRNA target site is over-represented in the transcripts encoding highly disordered proteins $(P<0.003)$ and under-represented in the transcripts encoding highly ordered proteins $(P$ $<$ o.00001) compared to all transcripts together (Figure S2a in Additional data file 1). A similar trend is observed when comparing the datasets of transcripts encoding ordered and

Table 3

\begin{tabular}{|c|c|c|c|c|c|}
\hline Expressor type & $\begin{array}{l}\text { Total transcripts } \\
\text { (frequency value) }\end{array}$ & $\begin{array}{c}\text { Percentage of transcripts } \\
\text { with different expression } \\
\text { profiles }\end{array}$ & $\begin{array}{c}\text { Transcripts with } \\
\text { miRNA (frequency } \\
\text { value) }\end{array}$ & $\begin{array}{c}\text { Transcripts with no } \\
\text { miRNA (frequency } \\
\text { value) }\end{array}$ & $\begin{array}{c}\text { Transcripts with } \\
\text { miRNA (\%) }\end{array}$ \\
\hline Tissue specific & $50(47)$ & 19.31 & 32 & 15 & 68.09 \\
\hline High & $43(4 I)$ & 16.60 & 27 & 14 & 65.85 \\
\hline Medium & $3 I(3 I)$ & 11.97 & 15 & 16 & 48.39 \\
\hline Constitutive & $4(1)$ & $\mathrm{I} .54$ & 0 & I & 0 \\
\hline Transient or low & |3| (129) & 50.58 & 62 & 67 & 48.06 \\
\hline Total & 259 & & & & \\
\hline
\end{tabular}




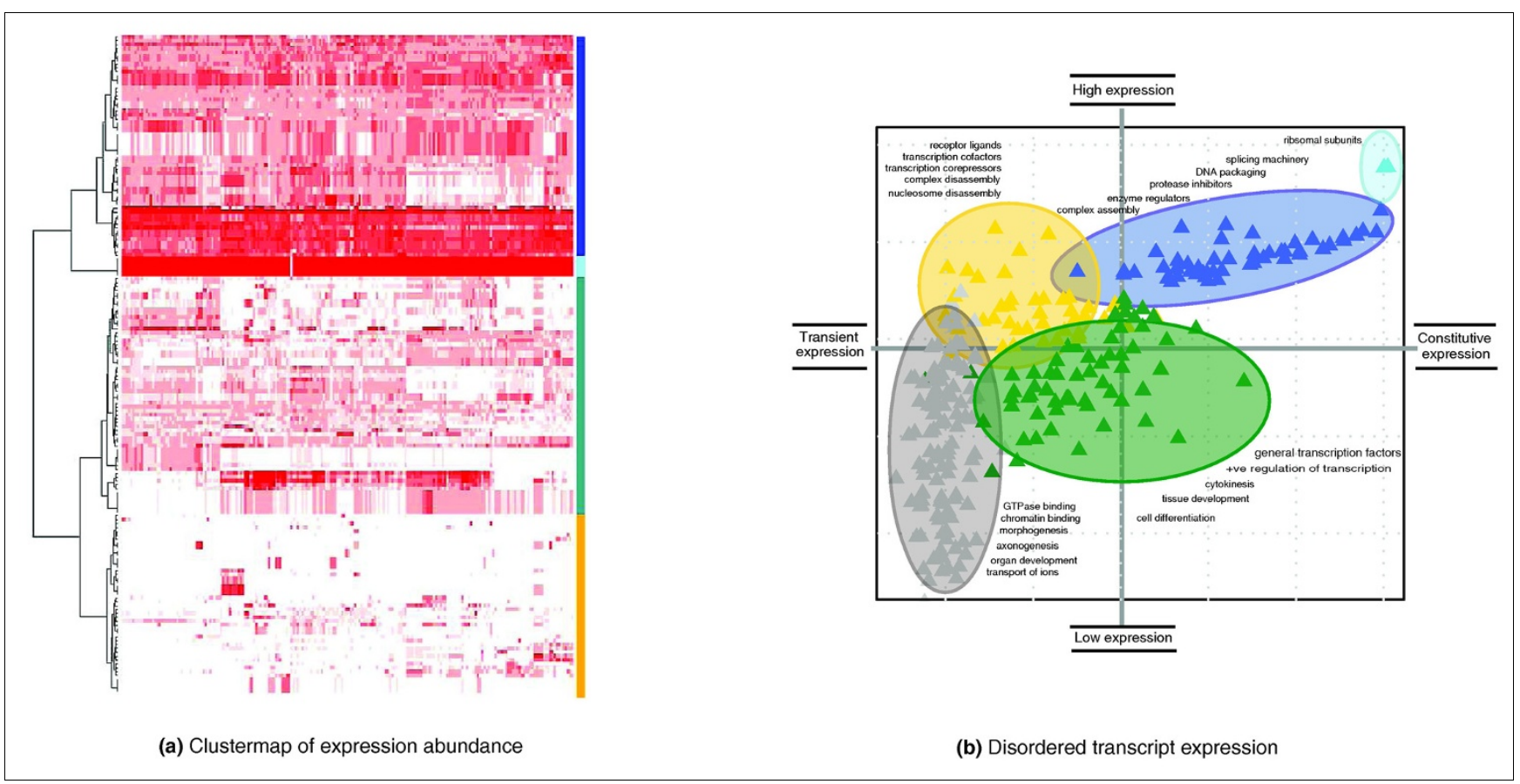

Figure 3

A summary of expression profiles for the highly disordered proteins. (a) The heat map displays four distinct transcript groups; constitutively expressed ribosomal subunits (light blue), high expressors (dark blue), medium expressors (green) and tissue specific expressors (gold). The clustering method was Ward's hierarchical clustering using Euclidean distances calculated over the absolute expression data matrix. Red colors indicate significantly high expression values $(P<0.001)$ within a sample tissue or cell line. (b). Summary of expression-function trends for highly disordered transcripts. Log 10 of the number of tissues in which the transcript is expressed ( $x$-axis); $\log _{10}$ expression of the average magnitude of expression within each tissue ( $y$-axis). The points have been jittered for overlap using a normally distributed noise value of 0.05 on the $\log _{10}$ scale.

disordered proteins (Table 2); the proportion of the transcripts encoding disordered proteins that are predicted as miRNA targets is approximately twice that of the transcripts encoding ordered proteins (Figure S1c in Additional data file 1; Table 2). miRNA targets are over-represented in the transcripts encoding disordered proteins $(P<0.00001)$ and under-represented in the transcripts encoding ordered proteins $(P<0.00001)$ compared to all transcripts together (Figure S2b in Additional data file 1).

For the transcripts encoding the proteome, the percent likely to be targeted by miRNA ranges between $13.2 \%$ and $37.6 \%$ (Figure 2d; Table 2). The percentage of transcripts regulated by miRNA increases (approximately 8\%) with increasing percentage of protein disorder for the first three binned categories (Figure 2c; Table 2). The percent of predicted miRNA targets for transcripts remains high (35.1\%) for the $(60,80] \%$ disorder category and low (13.2\%) for the [80,100]\% disorder category. Consistently, the likely miRNA targets are underrepresented in the $[0,20] \%$ and $(80,100] \%$ disorder categories at $P<0.00004$ (Figure S2c in Additional data file 1) and over-represented in the remaining three classes $\left(P<5.8 \times 10^{-}\right.$ 7; Figure S2c in Additional data file 1).

Similar trends are obtained using the PicTar (4-Way and 5Way) software [43,46] (Figures 1d and 2d; Figure S1c in Addi- tional data file 1). The trends were not observed using mirBase [51] and this could be because this prediction algorithm is reported to have a higher false positive rate than the other two programs (PicTar and TargetScanS) [47,49,50]. Redundancy in the datasets makes very little difference to the outcome (Table $\mathrm{S}_{3}$ in Additional data file 2). For example, the proteome and the protein sets filtered for redundancy have very similar percentages of transcripts predicted as targets of miRNA (Table 2; Table S3 in Additional data file 2).

We investigated the patterns of the predicted miRNA targets in the transcripts for disordered proteins in relation to the different expression profiles (Figures 3 and 4 and Table 3). The probes on the microarray chip have a higher representation of predicted miRNA targets (38\%) in comparison with the transcriptome encoding the human proteome (26.45\%) (Table 2). We compared the protein coding transcripts for the five datasets (Figure 3) using the probes on the microarray chip as a universal protein baseline. The data from the constitutive group had too few data points from which to make inferences (Table 3 and Figures 3 and 4). The tissue-specific expressors (gold) and the high expressors (dark blue) have high expression levels. The main difference between the two classes is that the tissue-specific expressors (gold) have high expression in one or few tissues (Figure 3) and the high expressors (dark blue) have high expression in almost all tissues (Figure 
Table 4

Subsets of GO terms (biological process, molecular function and cellular component) over-represented for co-regulated transcripts encoding highly disordered proteins

\begin{tabular}{|c|c|c|c|}
\hline Term & Description & Disorder $(60,80] \%$ & Disorder $(80,100] \%$ \\
\hline [GO:0005769] & Early endosome & Down & Down \\
\hline [GO:0005770] & Late endosome & Down & Down \\
\hline [GO:0005838] & Proteasome regulatory particle & Down & Down \\
\hline [GO:0016272] & Prefoldin complex & Down & \\
\hline [GO:003I37I] & Ubiquitin conjugating enzyme complex & Down & \\
\hline [GO:0000I45] & Exocyst & Down & \\
\hline [GO:0000502] & Proteasome complex & Down & \\
\hline [GO:003299I] & Macromolecular complex & & Up \\
\hline [GO:0043234] & Protein complex & & $U_{p}$ \\
\hline [GO:0019872] & Small conjugating protein ligase activity & & Up \\
\hline [GO:0042803] & Protein homodimerization activity & & Up \\
\hline [GO:005II3I] & Chaperone-mediated protein complex assembly & & Up \\
\hline [GO:0008639] & Small protein conjugating enzyme activity & & Up \\
\hline [GO:0004842] & Ubiquitin-protein ligase activity & & Up \\
\hline [GO:0016874] & Ligase activity & & $U_{p}$ \\
\hline [GO:00065I2] & Ubiquitin cycle & & $U_{p}$ \\
\hline [GO:0004869] & Cysteine protease inhibitor activity & Up & Up \\
\hline [GO:0004866] & Endopeptidase inhibitor activity & Up & $U_{p}$ \\
\hline [GO:00304I4] & Protease inhibitor activity & $U_{p}$ & $U_{p}$ \\
\hline [GO:005I082] & Unfolded protein binding & Up & Up \\
\hline [GO:0046983] & Protein dimerization activity & Up & Up \\
\hline [GO:005|29I] & Protein hetero-oligomerization & $U_{p}$ & \\
\hline [GO:0007032] & Endosome organization and biogenesis & Up & \\
\hline [GO:0006983] & ER overload response & Up & \\
\hline [GO:005I087] & Chaperone binding & Up & \\
\hline [GO:003।579] & Lipid raft organization and biogenesis & Up & \\
\hline [GO:0016235] & Aggresome & Up & \\
\hline [GO:0016234] & Inclusion body & Up & \\
\hline [GO:0016926] & Protein desumoylation & $U_{p}$ & \\
\hline [GO:000858I] & Ubiquitin specific protease 5 activity & Up & \\
\hline [GO:0006622] & Protein targeting to lysosome & Up & \\
\hline [GO:0019783] & Small conjugating protein-specific protease activity & & Down \\
\hline [GO:0008219] & Cell death & & Down \\
\hline [GO:0007049] & Cell death & & Down \\
\hline [GO:005I603] & Proteolysis involved in cellular protein catabolic process & Down & Down \\
\hline [GO:000422I] & Ubiquitin thiolesterase activity & Down & Down \\
\hline [GO:0016197] & Endosome transport & Down & Down \\
\hline [GO:0016874] & Ligase activity & Down & Down \\
\hline [GO:0004843] & Ubiquitin-specific protease activity & Down & Down \\
\hline [GO:005I082] & Unfolded protein binding & Down & Down \\
\hline [GO:0000209] & Protein polyubiquitination & Down & Down \\
\hline [GO:00065II] & Ubiquitin-dependent protein catabolic process & Down & \\
\hline [GO:00065I2] & Ubiquitin cycle & Down & \\
\hline [GO:005I087] & Chaperone binding & Down & \\
\hline [GO:0030968] & Unfolded protein response & Down & \\
\hline [GO:0030I00] & Regulation of endocytosis & Down & \\
\hline [GO:0043488] & Regulation of mRNA stability & Down & \\
\hline [GO:003।396] & Regulation of protein ubiquitination & Down & \\
\hline
\end{tabular}

Up, up-regulation; down, down-regulation. 
3). These two groups characterized by high levels of gene expression have high percentages of transcripts predicted as miRNA targets (68.09\% and 65.85\%, respectively; Table 3 and Figure 4). The medium expressors (green) and the low or transient expressors (white) with more moderate levels of gene expression have lower percentages of predicted miRNA targeting (48.39\% and 48.06\%, respectively). These results suggest that the transcripts of disordered proteins with high levels of expression are more likely to be regulated by miRNA compared to those with moderate and low or transient expression. In addition, the transcripts of highly disordered proteins belonging to the four expression profiles (tissue-specific, high expressors, medium expressors and low or transient expressors) are more likely to be miRNA targets than the transcripts on the microarray chip (Figure 4b). This observation supports the trend observed previously (Table 2) that transcripts encoding disordered proteins are more likely to be targeted by miRNAs compared to protein coding transcripts in general (Figure 4; Figures S1c and S2c in Additional data file 1).

\section{Protein disorder and ubiquitination}

To our knowledge, this study presents the first estimate of the percentage of proteins of the human proteome with at least one predicted ubiquitination site and the percentage of residues predicted as ubiquitination sites. We predict that $70.71 \%$ of proteins have at least one ubiquitination site and $0.42 \%$ of amino acid residues in the proteome are ubiquitination sites.

The percentage of proteins predicted to contain at least one ubiquitination site and the percentage of residues predicted as ubiquitination sites are higher in disordered proteins compared to ordered proteins. Comparing the highly disordered proteins with the highly ordered proteins, we observe increases of $33.81 \%$ and $42.50 \%$ in the percentage of proteins possessing at least one ubiquitination site and the percentage of residues predicted to be ubiquitination sites, respectively (Figure 1e). The proteins possessing at least one ubiquitination site are slightly over-represented in the highly disordered proteins $(P<0.98$; Figure S3a in Additional data file 1$)$ and grossly under-represented in the highly ordered proteins $(P<$ $2.2 \times 10^{-16}$; Figure S3a in Additional data file 1). The first trend is not statistically significant. The predicted ubiquitination sites are over-represented in the highly disordered proteins $\left(P<2.2 \times 10^{-16}\right.$; Figure S4a in Additional data file 1$)$ and under-represented for the highly ordered proteins $(P<$ 0.002; Figure S4a in Additional data file 1). Comparing the disordered proteins with the ordered proteins, we observe increases of $33.57 \%$ and $12.8 \%$ in the percentage of proteins possessing at least one ubiquitination site and the percentage of residues predicted to be ubiquitination sites, respectively (Figure S1d in Additional data file 1). Proteins with one or more predicted ubiquitination sites are over-represented in the disordered datasets $\left(P<2.2 \times 10^{-16}\right.$; Figure S3b in Additional data file 1 ) and under-represented in the ordered proteins $\left(P<2.2 \times 10^{-16}\right.$; Figure S3b in Additional data file 1$)$. A

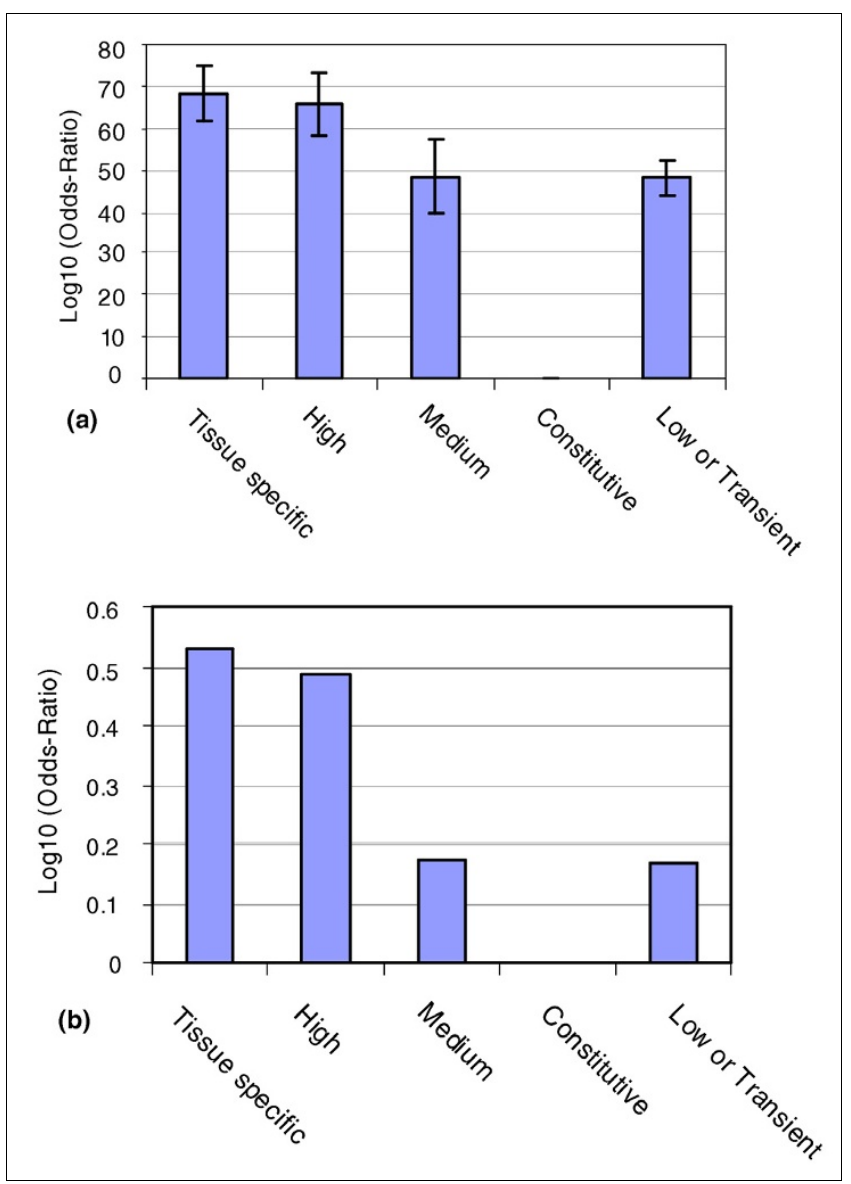

Figure 4

Summary of transcripts encoding highly disordered proteins as putative miRNA targets associated with expression profiles. (a) The percentage of the transcripts as predicted targets of miRNA ( $y$-axis) versus the different datasets (x-axis) that comprise transcripts with different patterns of gene expression (Table 3 ). The error bars represent the confidence in the percent value according to different sample sizes for the different groups. (b) The $\log _{10}$ odds-ratio (y-axis) discriminates categories as under-and over-represented in relation to being a predicted miRNA target.

similar trend is obtained for the percentage of residues predicted as ubiquitination sites.

The relationship between the percentage of proteins with at least one ubiquitination site and the percentage of protein disorder is complex and non-linear, while the percentage of residues predicted as ubiquitination sites and the percentage of protein disorder are positively correlated. The percentage of proteins predicted to have a ubiquitination site increases with the percentage of protein disorder for the first three disorder categories (Figure 2e). The percentage of proteins predicted to have a ubiquitination site remains high at $74.3 \%$ for the $(60,80] \%$ disorder class and then drops significantly to $55.8 \%$ for the $(80,100] \%$ disorder category. This is consistent with proteins with one or more predicted ubiquitination sites being over-represented in the $(20,40] \%,(40,60] \%$ and $(60,80] \%$ disorder categories $\left(P<0.04\right.$; Figure $S_{3 c}$ in Addi- 
tional data file 1) and under-represented in the [0,20]\% and $(80,100] \%$ disorder categories $(P<0.00005$; Figure S3c in Additional data file 1). On examination of the second ubiquitination descriptor, a different trend is observed; the percentage of residues predicted as ubiquitination sites increases as the percentage of protein disorder increases, illustrating a strong positive correlation between the two variables (Figure 2e). Proteins with one or more predicted ubiquitination sites are under-represented in the [0,20]\% disorder category and over-represented in the remaining four disorder classes $(P<$ $2.2 \times 10^{-16}$; Figure S4c in Additional data file 1).

As lysine is over-represented in disordered regions [1,76,77], we investigated the percentage of residues predicted as ubiquitination sites in relation to the percentageof protein disorder, taking into account lysine residue biases (Figure $\mathrm{S}_{5} \mathrm{a}$ in Additional data file 1). First, we calculated a correlation coefficient for the percentage of predicted ubiquitination sites and the percentage of lysine composition for the five disorder categories and obtained a strong positive correlation $(\mathrm{R}=$ 0.844772 ). Second, we normalized the number of predicted ubiquitination sites with respect to the number of lysines for each dataset. The trends observed for the percentage of predicted ubiquitination sites normalized for lysine frequency and disorder are similar to those obtained with the percentage of predicted ubiquitin sites and disorder ignoring lysine biases (Figure $\mathrm{S}_{5} \mathrm{~b}$ in Additional data file 1). Comparing the disorder categories with the order categories, the calculations normalized using the lysine frequency result in differences that are smaller in magnitude. For example, comparing the highly disordered proteins with the highly ordered proteins, an increase of $23.5 \%$ is observed instead of $42.5 \%$, and comparing the disordered proteins with the ordered proteins, an increase of $4.4 \%$ is observed instead of $12.8 \%$.

\section{Discussion}

This is the first analysis presenting a comprehensive and systematic study of gene expression levels, mRNA decay rates, miRNA targeting and ubiquitination in association with transcripts encoding protein disorder in humans. Using the human proteome and transcriptome, we set out to elucidate novel insights into the regulation of disordered proteins. This aim was achieved and we discuss our findings in the following sections.

\section{Protein disorder and gene expression}

On consideration of the gene expression levels for transcripts encoding disorder in proteins, two main trends emerge. Firstly, on average, the transcripts encoding disordered proteins are expressed to a significantly lower extent than those encoding ordered proteins. This suggests that, typically, the cell has evolved regulatory mechanisms to ensure that it does not have a large proportion of highly expressed transcripts encoding high amounts of protein disorder. Secondly, for the highly disordered proteins, there are five broad classes of gene expression patterns observed. These are constitutive expressors, high expressors, medium expressors, tissue-specific expressors and low or transient expressors. The constitutive expressors represent the transcripts that are constitutively and highly expressed across all samples. The high expressors contain protease inhibitors (specifically cysteine proteases) and enzyme regulatory functions. These proteins function in order to prevent their enzyme counterparts from cleaving peptide bonds; it is thus expected that their expression levels should remain relatively high in unstimulated tissue samples. This group is also enriched in functions directly related to splicing machinery, DNA packaging, nucleosome assembly and the components involved in DNA-protein and protein-protein complex assembly. In the medium expressor group, the transcription factors are predominantly positive regulators of transcription and are involved in muscle, cellular differentiation and general tissue development processes. The tissue-specific expressors are enriched in the transcription factors that target the nuclear hormone receptors and the ligands that are coordinately regulated with their receptor binding partners. The tissue-specific expressors are predominantly negative regulators of cell organization and promote complex disassembly and DNA unwinding and replication.

These novel observations have high biological relevance. Disordered proteins have important roles in the cell [1-31]; naturally they have to be expressed to carry out their specified function, but high levels of highly disordered proteins in the cell can cause a major problem through protein misfolding, misidentification and mis-signaling [24]. Our analysis suggests that one of the ways in which the cell keeps the level of highly disordered proteins under control is to keep the expression levels of transcripts encoding them low.

A recent study by Paliy and colleagues [78] claims that protein disorder is weakly positively correlated with gene expression in Eschericha coli. In our study, however, the trends between protein disorder and gene expression are complex and non-linear. Differences exist between the trends observed in E. coli [78] and those we report for human data. These differences are attributed to the differences in the methodologies (such as the disorder prediction methods and the definition of gene expression levels) and the consideration of species from disparate taxonomical classes. It is widely accepted that $E$. coli (a prokaryote) has about $5 \%$ of proteins that contain disordered regions whilst human (a eukaryote) has approximately 30\% [6]. Paliy et al. [78] examined the highly expressed transcripts for proteins possessing high levels of predicted disorder in a prokaryote (E. coli). The types of genes that fall into this category encode RNA and protein chaperones, protein carriers, transcriptional and translational regulators and multi-enzyme complexes. Some of the genes are found only in prokaryotes - these include the peptidoglycan-associated lipoprotein and the glycine cleavage Complex H protein [78] - whilst other genes exist in all taxo- 
nomical classes and some of these are identified in our study (for example, the ribosomal proteins and the translational initiation factor). The products of these transcripts whilst highly disordered are required by all cell types and by all species (prokaryote or eukaryote) and the transcripts are constitutively expressed at high levels. In contrast to the $E$. coli study [78], we find distinct transcripts encoding highly disordered proteins that are low or transiently expressed. These proteins are predominantly transcription factors or activators of transcription involved in developmental processes specific to complex higher organisms (Figure 3).

\section{Protein disorder, mRNA decay rates and protein stability indices}

We examined trends between mRNA decay rates and amounts of disordered residues, and between protein halflives and frequency of disordered residues. The mRNA decay rates for the transcripts encoding highly disordered proteins are more than twice that observed for the transcripts encoding highly ordered proteins. Large quantities of proteins containing high levels of disordered residues can cause function problems for the cell [24] and a higher mRNA decay rate could indicate the necessity for removal of potentially problematic proteins. Our finding of a correlation between high mRNA degradation rates and disordered proteins would appear to be in agreement with this.

The correlation between increasing amounts of protein disorder and longer half-lives was not expected since intrinsically disordered sequences are known to be extremely susceptible to proteolytic degradation [79]. However, these results were consistent with findings reported in Yen et al. [75], who observed an enrichment of disorder promoting residues in more stable proteins. This may be a feature of the way in which stability was measured. Attachment of an amino-terminal GFPS tag to a protein in the global protein stability assay may interfere with cellular localization and the authors of this study recognize that the stability values tended only to be reliable for nuclear proteins. Additionally, it is likely that this tag affects correct folding of the protein and might obscure amino-terminal degradation signals (N-degrons), which are a major determinant of stability in eukaryotic sequences [8o]. Considering these features of the available dataset, it may be that proteins with longer half-lives are enriched in the set that coincided with our data. However, the occurrence of highly stable sequences with long half-lives observed within sequences containing between $80 \%$ and $100 \%$ of disordered residues correlates well with the hypothesis that highly disordered proteins exist as complexes in vivo. A similar conclusion was drawn in the global protein stability profiling study where Yen et al. suggest that this mechanism constitutes a protection mechanism from cellular protein degradation machinery [75].

Recent work on protein disorder $[81,82]$ arrived at similar conclusions to our study. They suggested that certain disor- dered proteins may be required to remain in the cell for long periods of time, and thus need to avoid the degradation process. They suggest that such avoidances are evident by an increase in protein stability for some disordered proteins. Separately, they also found a correlation between decay rates and mRNA stability for disordered proteins, in agreement with our analysis.

\section{Protein disorder and miRNA targets}

We find a significantly higher level of predicted miRNA regulation of the transcripts encoding highly disordered proteins compared with the transcripts encoding highly ordered proteins. The predicted levels of miRNA regulation of the transcripts encoding highly disordered proteins are twice that observed for the transcripts encoding highly ordered proteins. Over one-third of the transcripts encoding disordered proteins are predicted to be regulated by miRNA. One-fifth of the transcripts encoding ordered proteins are predicted to be miRNA targets. Furthermore, miRNA-regulated gene expression is over-represented in transcripts encoding disordered proteins and under-represented in the transcripts coding for ordered proteins. These trends take into account $99 \%$ of the transcriptome encoding disordered proteins (that is, excluding the $(80,100] \%$ disorder category). We find that the transcripts of highly disordered proteins with high levels of expression are more likely to be affected by miRNA compared to those with moderate and low or transient expression. We provide strong evidence for miRNA regulation being particularly important for transcripts encoding disordered proteins. The observations make sense in a biological context. Typically, if a protein has high proportions of disorder, it is rapidly degraded in the cell [32]. For the cell, it would make economic sense to have an analogous system in place to handle the flagging up and degradation of the corresponding mRNA at the transcriptome level. This increased likelihood of miRNA binding to mRNA molecules that encode disordered proteins would regulate the gene expression of the mRNA molecule and prevent undesirable and wasteful translation of proteins no longer required by the cell.

\section{Protein disorder and ubiquitination}

The percentage of residues predicted as possible ubiquitination sites increases with increasing amounts of disorder. Interestingly, the relationship between these two properties is linear and positive. The trend between the percentage of proteins predicted to have one or more ubiquitination sites and disorder is more complex. The $(80,100] \%$ category has the lowest proportion of proteins predicted to have one or more ubiquitination sites compared to the remaining four categories. This follows a similar trend observed for mRNA decay rates and predicted miRNA targeting. This suggests that a significantly lower proportion of these proteins are likely not to be down-regulated by ubiquitination. This supports the observation described earlier that a significant proportion of highly disordered proteins is required to be expressed at high levels in all tissues or some tissues, and 
some are sometimes constitutively expressed. If high proportions of these highly disordered proteins and their corresponding transcripts did have positive signals for targeted degradation, this could adversely affect fitness. Additionally, highly disordered proteins with one or more predicted ubiquitination sites that are not constitutively or highly expressed may have a higher chance of being removed from the cell, as they are likely to have a higher density of ubiquitination sites. The $(80,100] \%$ disorder category is most likely to have the highest density of ubiquitination sites (Figure 2e).

\section{Protein disorder in relation to the five properties studied}

The increase in the decay rate of the transcripts encoding disordered proteins is likely attributable, in part, to the increase in predicted miRNA regulation. The transcripts encoding disordered proteins are targeted to a higher extent by miRNA compared to the transcripts encoding ordered proteins. This will result in the down-regulation of gene expression. The absolute gene expression levels and the predicted miRNA regulation are anti-correlated. The overall decrease in the gene expression of the transcripts encoding disordered proteins is likely attributable, in part, to the increased miRNA targeting that results in the down-regulation of these transcripts.

On the one hand, the majority of the disordered proteins have evolved with higher mRNA decay rates, higher levels of miRNA targeting, and higher levels of ubiquitination, which overall result in lower gene expression levels and protein levels for a high proportion of these disordered proteins compared to the ordered proteins. On the other hand, it is shown that for a significant proportion of highly disordered proteins, the converse is true. For the $(80,100] \%$ disorder class there is a decrease in mRNA decay rates, lower proportions of miRNA targeting and lower proportions of proteins being targeting for ubiquitination. These properties play a role in the high levels of gene expression observed in the highly disordered proteins compared to proteins with less disorder. The regulation of disordered proteins is affected by the various factors studied, and the relationships between these properties and protein disorder are inter-related, non-linear and complex.

Chen et al. [83] performed a structural biology analysis for the purpose of studying associations between structural vulnerability and co-expression in yeast and human. They claim that structural vulnerability (structural disorder) affects gene co-expression in a quantifiable manner [83]. In their study, they consider post-transcriptional regulation of transcripts of highly vulnerable proteins and find that $45 \%$ of human genes are predicted to have a least one miRNA target site compared to $82.9 \%$ of extremely vulnerable genes (87 out of 105) [83]. The mean number of miRNA target sites is 2.66 for human genes and 6.01 for vulnerable genes [83]. They show that vulnerability (disorder) requires significant additional regulation at the post-transcriptional level. This is an observation also made in our study; however, our miRNA study provides a more comprehensive analysis. For example, we investigate three different views of disorder. The first definition divides the transcriptome into transcripts encoding disordered proteins or ordered proteins. Second, transcripts encoding highly disordered or highly ordered proteins are considered. Third, we examined miRNA targeting of mRNAs encoding proteins with different percentages of disordered residues. Our study therefore provides more information in relation to disordered and highly disordered proteins. For example, we find that transcripts encoding disordered proteins are more likely to be targeted by miRNA than the transcripts encoding highly disordered proteins (Figures 1d and 2d; Figure S1c in Additional data file 1). Additionally, the method and the materials of Chen et al. [83] provide an estimate of miRNA targeting higher than our analysis and higher than estimates in other studies $[39,45]$. Our overall estimate of $26.5 \%$ for miRNA targeting agrees well with other estimates for miRNA targeting of protein coding transcripts $[39,45]$. miRNAs are shown to preferentially target genes with high transcriptional regulation complexity [84], and those involved in cellular signaling [85] and protein-protein interaction networks [86]. Functional properties such as transcriptional regulation, signaling and protein-protein interactions are associated with disordered proteins $[1,2,5-9,78,83]$. Our study highlights the association between predicted miRNA targets and protein disorder in a general way.

Mono-ubiquitination rather than polyubiquitination is the prevalent signal in intranuclear trafficking and triggers the first step of endocytosis [53,87-89]. The transcripts relating to polyubiquitination GO categories are down-co-regulated with highly disordered proteins (Table 4). Our results show that expression levels for the transcripts encoding highly disordered proteins are anti-correlated with transcripts involved in proteolysis and ubiquitin-dependent cellular catabolism. The expression levels for the transcripts encoding highly disordered proteins are positively correlated with proteolytic inhibitors. Whilst post-translational modification of proteins by ubiquitin is a key regulatory event, de-ubiquitination proteases, the enzymes that remove and process ubiquitin from proteins, are known to be functionally important [90]. Many of the de-ubiquitination proteases are cysteine proteases [90]. Transcripts coding for cysteine protease inhibitors, endopeptidase inhibitors and protease inhibitors are up-coregulated with highly disordered protein transcripts (Table 4), which may suggest that protease enzymes involved in deubiquitination are not expressed when the expression of disordered protein transcripts is high. We hypothesize that if a highly disordered protein has a ubiquitin tag, the function of this tag is more likely to be brought to fruition [87,90]. Highly disordered proteins with one or more predicted ubiquitination sites that are not constitutively or highly expressed may have a higher chance of being removed from the cell, as they are likely to have a higher density of ubiquitination sites. The $(80,100] \%$ disorder category is likely to have the highest density of ubiquitination sites (Figure 2e). Since ubiquitination is 
a feature of many biological processes [87-89] the presence of these ubiquitin target sites may also be implicated in protein transport between membrane components, possibly serving as a sorting signal and/or a regulatory signal for internalization into the endocytic pathways.

Transcripts associated with GO terms involved with protein complex formation are co-expressed with transcripts of highly disordered proteins (Table 4). Transcripts belonging to the $(60,80] \%$ disorder class are co-expressed with transcripts involved in heterodimeric complex assembly whilst those that belong to the $(80,100] \%$ class are co-expressed with those transcripts involved in homodimeric complex assembly. Several disordered proteins are known to be involved in protein cellular complexes [5,78,83], providing support for this finding. Transcriptome and interactome studies are known to provide complementary results [91,92]. The results indicating that chaperone binding correlates with highly disordered proteins (Table 4) are not conclusive and this is supported by a recent analysis [93]; it is hypothesized that disordered proteins that bind chaperones do so to avoid aggregation and assist in complex assembly [94-97]. Expression levels for transcripts involved in catabolic processes are down co-regulated with expression of transcripts encoding disordered proteins, that is expression levels of these transcript groups are simultaneously low (Table 4). This suggests that there is a reduction in the catabolism of biopolymers (such as proteins) and that the transcripts encoding highly disordered proteins and the resulting protein product remain in the cell longer to carry out function. The gene expression measurements were taken from 200 normal, 'resting' and un-stimulated tissues and this could partly explain the down-regulation of catabolic processes as well as other observations.

\section{Conclusions}

Our results suggest that the enrichment of miRNA targeting signals and ubiquitination signals may help prevent the accumulation of disordered proteins and their transcripts in the cell. Unexpectedly, for a proportion of highly disordered proteins, all four of these trends were reversed. The highly disordered proteins that are constitutively and/or highly expressed are shown to have low levels of estimated miRNA targeting, ubiquitination and lower mRNA decay rates, suggesting mechanisms by which highly disordered proteins can escape rapid degradation to allow them to successfully carry out their function. We conclude that the results of our study can serve as a baseline for characterizing the steady state abundance of disordered transcripts in normal tissues and cell lines as well as providing insights into how disordered transcripts might be regulated. These results can be used in future to compare disease states to normal states to identify disordered proteins, transcripts and modes of regulation that could be targets for therapeutic intervention in disease. Our study provides a better understanding of the regulation of transcripts for disordered proteins and some insights into the cel- lular regulatory mechanisms of key proteins that are likely to be involved in disease states.

\section{Materials and methods Disorder prediction in the human proteome}

Human protein sequences were obtained from the Ensembl FTP website (Assembly version 35). Disorder predictions were carried out using DISOPRED2 [6] with a $2 \%$ false positive rate. Our definition of a disordered protein states that it must contain at least one region of 30 contiguous disordered residues. This cut-off was based on previous work [98]. Two or more disordered regions separated by a number of ordered residues were considered distinct disordered regions. Using MEMSAT3 [99] and PFILT [100], we filtered our datasets for transmembrane and coiled-coil regions. This ensured a low false positive rate in our disorder predictions. An ordered protein is defined as one that has no disordered regions; that is, it does not contain a contiguous region comprising 30 or more predicted disordered residues. Using these definitions [98], proteins were classed as being ordered or disordered (Table 2).

A second classification scheme was devised to generate protein datasets reflective of high order and high disorder. To achieve this, we divided the proteome into 3 groups: those containing less than 10 residues of disorder; those containing $60 \%$ or more disordered residues; and those not fitting within either group. For the highly ordered protein dataset (Table 2), we chose a lower end cut-off of less than ten residues of disorder to allow for false positive disordered residues. For the highly disordered proteins (Table 2), an upper cut-off of $60 \%$ was used to ensure that this group contained genuine high levels of disorder in proteins. A third scheme to classify disorder in proteins was based on the percentage of disordered residues in the protein. The percentage of disordered residues in the protein datasets were binned (Table 2). The first bin [0,20]\% has from $0 \%$ (inclusive) to $20 \%$ (inclusive) residues disordered; the second bin $(20,40] \%$ has $>20 \%$ and $\leq 40 \%$ residues disordered and so on until the final bin $(80,100] \%$, which has $>80 \%$ and $\leq 100 \%$ residues disordered.

\section{Protein disorder and gene expression}

Microarray data pre-processing, normalization and summarization We combined and integrated 207 normal tissue and cell line samples at the probe-level from microarray datasets (Table 1) downloaded from the Gene Expression Omnibus (GEO) database [101]. The Novartis gene expression atlas includes 79 samples, each having two replicates. Seven samples (cancer tissues and an unknown tissue type) were excluded (Table 1). Each experimental sample was adjusted for background using the GCRMA algorithm [102] and quantile normalized using a common reference distribution constructed from 50 selected and maximally varying U133a chip samples. Summarization was carried out on a per-transcript level using the Ensembl transcript hs133ahsenst, hs133ahsenstcdf and 
hs133ahsenstprobe custom cdf environments [103]. Sample $\mathrm{R}$ code [104] for the procedure can be found online [105].

\section{Comparison of absolute transcript abundance}

The transcripts identified on the microarray were divided into two distinct groups: those encoding highly disordered proteins (346) and those encoding highly ordered proteins $(2,127)$. The average transcript abundance in each tissue was calculated and compared between the two groups. The same was carried out for disordered $(10,681)$ and ordered $(4,883)$ categories.

\section{Evaluating significant absolute transcript expressions}

Significance values were calculated for absolute transcript abundances within sample tissues and cell lines using $\mathrm{Z}$ scores to identify outliers. The null hypothesis that the transcript was not significantly expressed in the sample was rejected at $P$-values of $<0.05$.

\section{Evaluating significant expression correlations}

Relative transcript abundances were obtained by double mean centering the absolute transcript levels across the tissues and between the probe-sets. The mean expression for each probe-set was weighted according to the number of replicates in each tissue group. Weights were calculated such that the contribution of sample replicates for the same tissue or cell line summed to 1 . Co-expression of the transcript pairs was then evaluated using a weighted correlation coefficient. Significance of correlation was evaluated explicitly by applying a $\mathrm{Z}$ score transform to the distribution of Pearson correlation coefficients. Transcripts were considered to be significantly correlated and consequently co-expressed at $P$ values of $<0.01$. This significance threshold corresponded to Pearson's correlation values of 0.749 and -0.729 at the upper and lower distribution tails, respectively (Figure S6 in Additional data file 1).

\section{Gene Ontology analysis}

GO annotations were downloaded from Ensembl BioMart [106] for BP and MF transcripts. To robustly identify overrepresentation of functions in the sets of transcripts co-regulated with disordered transcripts, a two step statistical testing procedure was used. First, multiple hypergeometric testing was performed to identify functions enriched within a set of co-regulated transcripts for a given disordered transcript. Prior probabilities for each function class were determined by the observed frequencies across all transcripts. The null hypothesis was rejected at $P$-values below 0.05 controlling the false discovery rate at $5 \%$. Subsequently binomial testing was performed (assuming replacement) to model the enrichment of functions common to groups of co-regulated transcripts. The prior probability of enrichment for a function class between multiple sets of co-regulated transcripts was determined by evaluating the frequency of positive outcomes resulting from the hypergeometric test over all co-regulated transcripts. The false discovery rate for group-wise functional enrichment was controlled at $1 \%$.

\section{Protein disorder, mRNA decay rates and protein stability indices}

In previous work, Yang et al. [33] measured the mRNA decay rates of 5,245 human transcripts. The mapping of the EMBL (GenBank/DBJ) identifiers for these mRNA transcripts [33] to the Ensembl protein identifiers of our protein datasets was facilitated by the use of Biomart [106]. Using their EMBL (GenBank/DBJ) identifiers, we mapped the decay rates to the Ensembl protein identifiers of our dataset of human proteins. This gave a dataset of 3,839 proteins, each of which has an associated experimentally determined mRNA decay rate. We separated our 3,839 protein dataset into 3 groups; highly disordered (74 proteins); highly ordered (536 proteins); and the remainder (3,229 proteins).

Recent work by Yen et al. [75] reported half-life protein stability measures for more than 8,00o human proteins using their global protein stability assay. This study is one of the most comprehensive studies of in vivo stability measured for proteins in complex cellular mixtures. We mapped these stability measures to our disorder protein dataset, which resulted in a set of 6,886 proteins. We separated this dataset into three groups, as described above. This resulted in 179 highly disordered proteins, 1,396 highly ordered proteins and 5,311 remaining proteins.

\section{Protein disorder and miRNA targets}

The predicted mRNA targets of mammalian miRNAs were downloaded from the TargetScanS website [44]. The dataset downloaded (release 4.2; April 2008) contains 218,298 mRNA target predictions. The predictions were performed using TargetScans [44]. From this dataset, 7,928 unique genes (HUGO identifiers provided) were predicted to be targets of miRNA. The HUGO identifiers [107] for the mRNA genes were extracted into a gene list. An analysis pipeline was developed to establish whether a correlation exists between the disordered proteins and the transcripts targeted by miRNA. The first part uses the Ensembl database and the PERL API [108] to map the HUGO identifiers to Ensembl identifiers (the gene, the transcript and the protein) and extract the associated translated protein sequences. We identified 15,954 transcripts for the 7,928 HUGO gene identifiers. The second part categorizes the protein datasets based on the amounts of disorder (Table 2). With each protein category, a protein list is derived and protein sequence dataset is created. The third part compares each protein dataset with the translated products of the mRNA targets regulated by miRNAs by tallying identical protein sequences derived from the 15,954 transcripts and the transcripts from each disorder category. For a given dataset (Table 2), to calculate the percentage of protein transcripts that are predicted miRNA targets, the number of matches between the two datasets was divided by the total number of proteins in the disordered protein cate- 
gory under consideration and this fraction was multiplied by 100. Fisher's exact tests were carried out in R [109] to identify groups of transcripts that were enriched in miRNA target sites.

\section{Protein disorder and ubiquitination}

Ubiquitin targeting signal sites were predicted for the peptides encoded by the Ensembl transcripts using a neural network-based predictor. Our predictor uses a single hiddenlayer back-propagation network trained to recognize features of ubiquitin targeting signals over a sliding window of 21 amino acids in the target sequence. The network was trained using a balanced dataset of Swiss-Prot annotated ubiquitination sites [110] and rigorously cross-validated using a jack-knife leave-one-out approach. The performance of our predictor on both the Swiss-Prot training data and a non-redundant experimentally defined dataset [110] was also determined (Figure S7 in Additional data file1). Predictions were only reported at a false discovery rate of $<5 \%$ estimated from our model. Although no ubiquitination predictors were available when we carried out this study, the Ubi-pred tool [111] has subsequently been published. This tool obtains a final area under the curve (AUC) [112] of 0.85, sensitivity of 70.86, specificity of 0.954 and a Matthews correlation coefficient of 0.69 compared to our own ubiquitination predictor, which obtains a final AUC of 0.88 , sensitivity of 83.44, specificity of 85.43 and a Matthews correlation coefficient of 0.69 . These statistics provided the justification for using our own ubiquitination prediction tool over Ubi-pred [111] due to the observed lower false positive rate. The percentage accuracy is defined here as $100 \%$ - Percentage of errors and the AUC is the area under the receiver operating characteristic (ROC) curve (a standard score for prediction algorithms between $\mathrm{O}$ and 1).

As an independent test of our ubiquitin site prediction algorithm, we scanned a dataset of putative and experimentally determined ligase target sequences [113] for the presence of ubiquitin modification sites (Table $\mathrm{S}_{4}$ in Additional data file 2). In total, $72.6 \%$ of sequences (257 of 345 ) were predicted to contain at least 1 modification site. This demonstrates that our in silico predictions are in good agreement with independent experimental outcomes.

\section{Abbreviations}

AUC: area under the curve; BP: biological process; GEO: Gene Expression Omnibus; GO: Gene Ontology; MF: molecular function; miRNA: microRNA.

\section{Authors' contributions}

YKE carried out the miRNA and ubiquitin target analysis and drafted the major part of the manuscript. AEL carried out the microarray analysis, ubiquitin predictions and function analysis and assisted in all other aspects of the work. MP carried out the analysis of decay rates and protein stability. DTJ provided the ubiquitin site prediction algorithm. DTJ and AEL conceived of the study and participated in its design and coordination. All authors contributed to and approved the final manuscript.

\section{Additional data files}

The following additional data are available with the online version of this paper: supplementary Figures S1 to S7 (Additional data file 1); supplementary Tables S1 to S4 (Additional data file 2).

\section{Acknowledgements}

One of the authors, YJKE, was financially supported by the Wellcome Trust Functional Genomics Initiative on Stem Cells (grant number GR066745MA). The funders had no role in study design, data collection and analysis, decision to publish, or preparation of the manuscript. We acknowledge technical support for provision of computer services that enabled us to complete this work and Dr Kevin Bryson for helpful discussions.

\section{References}

I. Dunker AK, Lawson JD, Brown C], Williams RM, Romero P, Oh JS, Oldfield CJ, Campen AM, Ratliff CM, Hipps KW, Ausio J, Nissen MS, Reeves R, Kang C, Kissinger CR, Bailey RW, Griswold MD, Chiu W, Garner EC, Obradovic Z: Intrinsically disordered protein. J Mol Graph Model 200I, 19:26-59.

2. Dunker AK, Brown CJ, Lawson JD, lakoucheva LM, Obradovic Z Intrinsic disorder and protein function. Biochemistry 2002, 4I:6573-6582.

3. Dunker AK, Cortese MS, Romero P, lakoucheva LM, Uversky VN: Flexible nets. The roles of intrinsic disorder in protein interaction networks. FEBS J 2005, 272:5 I 29-5 I48.

4. Uversky VN, Oldfield C], Dunker AK: Showing your ID: intrinsic disorder as an ID for recognition, regulation and cell signaling. J Mol Recognit 2005, 18:343-384.

5. Hegyi H, Schad E, Tompa P: Structural disorder promotes assembly of protein complexes. BMC Struct Biol 2007, 7:65.

6. Ward J], Sodhi JS, McGuffin L], Buxton BF, Jones DT: Prediction and functional analysis of native disorder in proteins from the three kingdoms of life. J Mol Biol 2004, 337:635-645.

7. Dyson HJ, Wright PE: Intrinsically unstructured proteins and their functions. Nat Rev Mol Cell Biol 2005, 6: 197-208.

8. Haynes C, Oldfield CJ, Ji F, Klitgord N, Cusick ME, Radivojac P, Uversky VN, Vidal M, lakoucheva LM: Intrinsic disorder is a common feature of hub proteins from four eukaryotic interactomes. PLoS Comput Biol 2006, 2: 100.

9. Lobley A, Swindells MB, Orengo CA, Jones DT: Inferring function using patterns of native disorder in proteins. PLoS Comput Biol 2007, 3:el62.

10. Wright PE, Dyson HJ: Intrinsically unstructured proteins: reassessing the protein structure-function paradigm. J Mol Biol 1999, 293:321-331.

II. Uversky VN, Gillespie JR, Fink AL: Why are "natively unfolded" proteins unstructured under physiologic conditions? Proteins 2000, 4I:4I5-427.

12. Dunker $A K$, Obradovic $Z$ : The protein trinity - linking function and disorder. Nat Biotechnol 200I, 19:805-806.

13. Tompa P: Intrinsically unstructured proteins. Trends Biochem Sci 2002, 27:527-533.

14. Uversky VN: Natively unfolded proteins: a point where biology waits for physics. Protein Sci 2002, I I:739-756.

15. Uversky VN: Protein folding revisited. A polypeptide chain at the folding-misfolding-nonfolding cross-roads: which way to go? Cell Mol Life Sci 2003, 60:1852-187I.

16. Tompa $P$ : The interplay between structure and function in intrinsically unstructured proteins. FEBS Lett 2005, 579:3346-3354.

17. Xie H, Vucetic S, lakoucheva LM, Oldfield CJ, Dunker AK, Obradovic 
Z, Uversky VN: Functional anthology of intrinsic disorder. 3. Ligands, post-translational modifications, and diseases associated with intrinsically disordered proteins. J Proteome Res 2007, 6:1917-1932.

18. Vucetic S, Xie H, lakoucheva LM, Oldfield CJ, Dunker AK, Obradovic Z, Uversky VN: Functional anthology of intrinsic disorder. 2. Cellular components, domains, technical terms, developmental processes, and coding sequence diversities correlated with long disordered regions. J Proteome Res 2007, 6:1899-1916.

19. Xie H, Vucetic S, lakoucheva LM, Oldfield CJ, Dunker AK, Uversky VN, Obradovic Z: Functional anthology of intrinsic disorder. I. Biological processes and functions of proteins with long disordered regions. J Proteome Res 2007, 6:1882-1898.

20. Cortese MS, Uversky VN, Dunker AK: Intrinsic disorder in scaffold proteins: getting more from less. Prog Biophys Mol Biol 2008, 98:85-106.

21. lakoucheva LM, Brown CJ, Lawson JD, Obradovic Z, Dunker AK: Intrinsic disorder in cell-signaling and cancer-associated proteins. J Mol Biol 2002, 323:573-584.

22. Dunker AK, Obradovic Z, Romero P, Garner EC, Brown CJ: Intrinsic protein disorder in complete genomes. Genome Inform Ser Workshop Genome Inform 2000, I I:|6|-|7|

23. Oldfield CJ, Cheng Y, Cortese MS, Brown CJ, Uversky VN, Dunker AK: Comparing and combining predictors of mostly disordered proteins. Biochemistry 2005, 44:1989-2000.

24. Uversky VN, Oldfield CJ, Dunker AK: Intrinsically disordered proteins in human diseases: introducing the D2 concept. Annu Rev Biophys 2008, 37:2I5-246.

25. Weinreb PH, Zhen W, Poon AW, Conway KA, Lansbury PT Jr: NACP, a protein implicated in Alzheimer's disease and learning, is natively unfolded. Biochemistry 1996, 35:13709-13715.

26. Rochet JC, Lansbury PT Jr: Amyloid fibrillogenesis: themes and variations. Curr Opin Struct Biol 2000, 10:60-68.

27. Uversky VN: Alpha-synuclein misfolding and neurodegenerative diseases. Curr Protein Pept Sci 2008, 9:507-540.

28. Uversky VN: Amyloidogenesis of natively unfolded proteins. Curr Alzheimer Res 2008, 5:260-287.

29. Cheng Y, LeGall T, Oldfield CJ, Dunker AK, Uversky VN: Abundance of intrinsic disorder in protein associated with cardiovascular disease. Biochemistry 2006, 45:10448-10460.

30. Uversky VN, Roman A, Oldfield CJ, Dunker AK: Protein intrinsic disorder and human papillomaviruses: increased amount of disorder in E6 and E7 oncoproteins from high risk HPVs. J Proteome Res 2006, 5: 1829-1842.

31. Galzitskaya OV, Garbuzynskiy SO, Lobanov MY: Prediction of amyloidogenic and disordered regions in protein chains. PLOS Comput Biol 2006, 2:el 77.

32. Goldberg AL: Protein degradation and protection against misfolded or damaged proteins. Nature 2003, 426:895-899.

33. Yang E, van Nimwegen E, Zavolan M, Rajewsky N, Schroeder M, Magnasco M, Darnell JE Jr: Decay rates of human mRNAs: correlation with functional characteristics and sequence attributes. Genome Res 2003, 13:1863-1872.

34. Raghavan A, Bohjanen PR: Microarray-based analyses of mRNA decay in the regulation of mammalian gene expression. Brief Funct Genomic Proteomic 2004, 3: I 12-124.

35. Tompa P, Prilusky J, Silman I, Sussman JL: Structural disorder serves as a weak signal for intracellular protein degradation. Proteins 2008, 71:903-909.

36. Orphanides $G$, Reinberg $D$ : A unified theory of gene expression. Cell 2002, I08:439-45I.

37. He L, Hannon GJ: MicroRNAs: small RNAs with a big role in gene regulation. Nat Rev Genet 2004, 5:522-53I.

38. Bartel DP: MicroRNAs: genomics, biogenesis, mechanism, and function. Cell 2004, I 1 6:28I-297.

39. Jackson RJ, Standart N: How do microRNAs regulate gene expression? SCi STKE 2007, 2007:rel

40. Lodish HF, Zhou B, Liu G, Chen CZ: Micromanagement of the immune system by microRNAs. Nat Rev Immunol 2008, 8:120-130.

41. Hobert O: Gene regulation by transcription factors and microRNAs. Science 2008, 319:1785-1786.

42. Ambros V: The functions of animal microRNAs. Nature 2004, 43I:350-355.

43. Krek A, Grün D, Poy MN, Wolf R, Rosenberg L, Epstein EJ, MacMenamin P, da Piedade I, Gunsalus KC, Stoffel M, Rajewsky N: Combi- natorial microRNA target predictions. Nat Genet 2005, 37:495-500

44. TargetScanS. [http://www.targetscan.org/]

45. Lewis BP, Burge CB, Bartel DP: Conserved seed pairing, often flanked by adenosines, indicates that thousands of human genes are microRNA targets. Cell 2005, 120:15-20.

46. Grün D, Wang YL, Langenberger D, Gunsalus KC, Rajewsky N: MicroRNA target predictions across seven Drosophila species and comparison to mammalian targets. PLoS Comput Biol 2005, I:el3.

47. Rajewsky N: microRNA target predictions in animals. Nat Genet 2006, 38(Suppl):S8-SI3.

48. Sethupathy P, Corda B, Hatzigeorgiou AG: TarBase: A comprehensive database of experimentally supported animal microRNA targets. RNA 2006, 1 2:192-197.

49. Sethupathy P, Megraw M, Hatzigeorgiou AG: A guide through present computational approaches for the identification of mammalian microRNA targets. Nat Methods 2006, 3:88I-886.

50. Mazière $P$, Enright AJ: Prediction of microRNA targets. Drug Discovery Today 2007, I 2:452-458.

5I. Griffiths-Jones S, Saini HK, van Dongen S, Enright AJ: miRBase: tools for microRNA genomics. Nucleic Acids Res 2008, 36:DI54-DI58.

52. Haglund $K$, Dikic I: Ubiquitylation and cell signaling. $E M B O J$ 2005, 24:3353-3359.

53. Welchman RL, Gordon C, Mayer RJ: Ubiquitin and ubiquitin-like proteins as multifunctional signals. Nat Rev Mol Cell Biol 2005, 6:599-609.

54. Pickart CM, Fushman D: Polyubiquitin chains: polymeric protein signals. Curr Opin Chem Biol 2004, 8:610-6I6.

55. Thrower JS, Hoffman L, Rechsteiner M, Pickart CM: Recognition of the polyubiquitin proteolytic signal. EMBO J 2000, 19:94-102.

56. Salghetti SE, Caudy AA, Chenoweth JG, Tansey WP: Regulation of transcriptional activation domain function by ubiquitin. Science 200I, 293:165I-1653.

57. Muratani M, Tansey WP: How the ubiquitin-proteasome system controls transcription. Nat Rev Mol Cell Biol 2003, 4:192-20I.

58. Lipford JR, Deshaies RJ: Diverse roles for ubiquitin-dependent proteolysis in transcriptional activation. Nat Cell Biol 2003, 5:845-850.

59. Molnár A, Schwach F, Studholme DJ, Thuenemann EC, Baulcombe DC: miRNAs control gene expression in the single-cell alga Chlamydomonas reinhardtii. Nature 2007, 447: I I 26-I I 29.

60. Zhao T, Li G, Mi S, Li S, Hannon GJ, Wang XJ, Qi Y: A complex system of small RNAs in the unicellular green alga Chlamydomonas reinhardtii. Genes Dev 2007, 21:1190-1203.

61. Ha BH, Kim EE: Structures of proteases for ubiqutin and ubiquitin-like modifiers. BMB Rep 2008, 41:435-443.

62. Calin GA, Croce CM: MicroRNA signatures in human cancers. Nat Rev Cancer 2006, 6:857-866.

63. Calin GA, Croce CM: MicroRNA-cancer connection: the beginning of a new tale. Cancer Res 2006, 66:7390-7394.

64. Hoeller D, Hecker CM, Dikic I: Ubiquitin and ubiquitin-like proteins in cancer pathogenesis. Nat Rev Cancer 2006, 6:776-788.

65. Bushati N, Cohen SM: MicroRNAs in neurodegeneration. Curr Opin Neurobiol 2008, 18:292-296.

66. Rubinsztein DC: The roles of intracellular protein-degradation pathways in neurodegeneration. Nature 2006, 443:780-786.

67. Scalbert E, Bril A: Implication of microRNAs in the cardiovascular system. Curr Opin Pharmacol 2008, 8: $|8|-\mid 88$.

68. Patterson C, Ike C, Willis PW 4th, Stouffer GA, Willis MS: The bitter end: the ubiquitin-proteasome system and cardiac dysfunction. Circulation 2007, I I 5: / 456-1463.

69. Mearini G, Schlossarek S, Willis MS, Carrier L: The ubiquitin-proteasome system in cardiac dysfunction. Biochim Biophys Acto 2008, 1782:749-763.

70. Lenburg ME, Liou LS, Gerry NP, Frampton GM, Cohen HT, Christman MF: Previously unidentified changes in renal cell carcinoma gene expression identified by parametric analysis of microarray data. BMC Cancer 2003, 3:31.

7I. Su AI, Wiltshire T, Batalov S, Lapp H, Ching KA, Block D, Zhang J, Soden R, Hayakawa M, Kreiman G, Cooke MP, Walker JR, Hogenesch JB: A gene atlas of the mouse and human protein-encoding transcriptomes. Proc Natl Acad Sci USA 2004, 10 I:6062-6067.

72. Ge X, Yamamoto S, Tsutsumi S, Midorikawa Y, Ihara S, Wang SM, Aburatani $\mathrm{H}$ : Interpreting expression profiles of cancers by genome-wide survey of breadth of expression in normal tissues. Genomics 2005, 86:|27-|4I. 
73. Cordero F, Botta M, Calogero RA: Microarray data analysis and mining approaches. Brief Funct Genomic Proteomic 2007, 6:265-28I.

74. Kilpinen S, Autio R, Ojala K, Iljin K, Bucher E, Sara H, Pisto T, Saarela M, Skotheim RI, Björkman M, Mpindi JP, Haapa-Paananen S, Vainio P, Edgren $\mathrm{H}$, Wolf $\mathrm{M}$, Astola J, Nees M, Hautaniemi S, Kallioniemi O: Systematic bioinformatic analysis of expression levels of I 7,330 human genes across 9,783 samples from I 75 types of healthy and pathological tissues. Genome Biol 2008, 9:R I39.

75. Yen HC, Xu Q, Chou DM, Zhao Z, Elledge SJ: Global protein stability profiling in mammalian cells. Science 2008, 322:918-923.

76. Romero P, Obradovic Z, Li X, Garner EC, Brown CJ, Dunker AK Sequence complexity of disordered protein. Proteins 200I, 42:38-48.

77. Vucetic S, Brown CJ, Dunker AK, Obradovic Z: Flavors of protein disorder. Proteins 2003, 52:573-584.

78. Paliy O, Gargac SM, Cheng Y, Uversky VN, Dunker AK: Protein disorder is positively correlated with gene expression in Escherichia coli. J Proteome Res 2008, 7:2234-2245.

79. Dunker K, Silman I, Uversky VN, Sussman JL: Function and structure of inherantly disordered proteins. Curr Opin Struct Biol 2008, I 8:756-764.

80. Mogka A, Schmidta R, Bukaua B: The $\mathbf{N}$-end rule pathway for regulated proteolysis: prokaryotic and eukaryotic strategies. Trends Cell Biol 2007, I 7:165-I72.

81. Gsponer J, Futschik ME, Teichmann SA, Babu MM: Tight regulation of unstructured proteins: from transcript synthesis to protein degradation. Science 2008, 322:1365-|368.

82. Uversky VN, Dunker AK: Biochemistry: Controlled chaos. Science 2008, 322:1340-134I.

83. Chen J, Liang H, Fernández A: Protein structure protection commits gene expression patterns. Genome Biol 2008, 9:RI07.

84. Cui Q, Yu Z, Pan Y, Purisima EO, Wang E: MicroRNAs preferentially target the genes with high transcriptional regulation complexity. Biochem Biophys Res Commun 2007, 352:733-738.

85. Cui Q, Yu Z, Purisima EO, Wang E: Principles of microRNA regulation of a human cellular signaling network. Mol Syst Biol 2006, 2:46.

86. Liang $\mathrm{H}$, Li WH: MicroRNA regulation of human protein protein interaction network. RNA 2007, I3:|402-| 408.

87. Mukhopadhyay D, Riezman H: Proteasome-independent functions of ubiquitin in endocytosis and signaling. Science 2007, 3 | 5:20|-205.

88. Mellman I, Nelson WJ: Coordinated protein sorting, targeting and distribution in polarized cells. Nat Rev Mol Cell Biol 2008, 9:833-845.

89. Hicke L, Dunn R: Regulation of membrane protein transport by ubiquitin and ubiquitin-binding proteins. Annu Rev Cell Dev Biol 2003, 19:141-172.

90. Nijman SM, Luna-Vargas MP, Velds A, Brummelkamp TR, Dirac AM, Sixma TK, Bernards R: A genomic and functional inventory of deubiquitinating enzymes. Cell 2005, I 23:773-786.

91. Ge H, Liu Z, Church GM, Vidal M: Correlation between transcriptome and interactome mapping data from Saccharomyces cerevisiae. Nat Genet 200I, 29:482-486.

92. Jansen R, Greenbaum D, Gerstein M: Relating whole-genome expression data with protein-protein interactions. Genome Res 2002, I 2:37-46.

93. Hegyi H, Tompa P: Intrinsically disordered proteins display no preference for chaperone binding in vivo. PLOS Comput Biol 2008, 4:el000017.

94. Kovacs D, Rakacs M, Agoston B, Lenkey K, Semrad K, Schroeder R, Tompa P: Janus chaperones: assistance of both RNA- and protein-folding by ribosomal proteins. FEBS Lett 2008, 583:88-92.

95. Fuxreiter M, Tompa P, Simon I, Uversky VN, Hansen JC, Asturias FJ: Malleable machines take shape in eukaryotic transcriptional regulation. Nat Chem Biol 2008, 4:728-737.

96. Tompa P, Fuxreiter M: Fuzzy complexes: polymorphism and structural disorder in protein-protein interactions. Trends Biochem Sci 2008, 33:2-8.

97. Dice JF: Chaperone-mediated autophagy. Autophagy 2007, 3:295-299.

98. Ward JJ, McGuffin LJ, Bryson K, Buxton BF, Jones DT: The DISOPRED server for the prediction of protein disorder. Bioinformatics 2004, 20:2138-2139.

99. Jones DT: Improving the accuracy of transmembrane protein topology prediction using evolutionary information. Bioinformatics 2007, 23:538-544.

100. Jones DT, Taylor WR, Thornton JM: A model recognition approach to the prediction of all-helical membrane protein structure and topology. Biochemistry 1994, 33:3038-3049.

I0I. Barrett T, Troup DB, Wilhite SE, Ledoux P, Rudnev D, Evangelista C, Kim IF, Soboleva A, Tomashevsky M, Edgar R: NCBI GEO: mining tens of millions of expression profiles - database and tools update. Nucleic Acids Res 2007, 35:D760-D765.

102. Wu Z, Irizarry RA: Preprocessing of oligonucleotide array data. Nat Biotechnol 2004, 22:656-658.

103. Dai M, Wang P, Boyd AD, Kostov G, Athey B, Jones EG, Bunney WE, Myers RM, Speed TP, Akil H, Watson SJ, Meng F: Evolving genel transcript definitions significantly alter the interpretation of GeneChip data. Nucleic Acids Res 2005, 33:e 175.

104. Gentleman RC, Carey VJ, Bates DM, Bolstad B, Dettling M, Dudoit S, Ellis B, Gautier L, Ge Y, Gentry J, Hornik K, Hothorn T, Huber W, lacus S, Irizarry R, Leisch F, Li C, Maechler M, Rossini AJ, Sawitzki G, Smith C, Smyth G, Tierney L, Yang JY, Zhang J: Bioconductor: open software development for computational biology and bioinformatics. Genome Biol 2004, 5:R80.

105. Sample $\mathbf{R}$ code for microarray pre-processing. [http://bio inf.cs.ucl.ac.uk/disorder_regulation/]

106. Kasprzyk A, Keefe D, Smedley D, London D, Spooner W, Melsopp C, Hammond M, Rocca-Serra P, Cox T, Birney E: EnsMart: a generic system for fast and flexible access to biological data. Genome Res 2004, I4:160-169.

107. Bruford EA, Lush MJ, Wright MW, Sneddon TP, Povey S, Birney E: The HGNC Database in 2008: a resource for the human genome. Nucleic Acids Res 2008, 36:D445-D448.

108. Flicek P, Aken BL, Beal K, Ballester B, Caccamo M, Chen Y, Clarke L, Coates G, Cunningham F, Cutts T, Down T, Dyer SC, Eyre T, Fitzgerald S, Fernandez-Banet J, Gräf S, Haider S, Hammond M, Holland R, Howe KL, Howe K, Johnson N, Jenkinson A, Kähäri A, Keefe D, Kokocinski F, Kulesha E, Lawson D, Longden I, Megy K, et al: Ensembl 2008. Nucleic Acids Res 2008, 36:D707-D7I4.

109. R: A Language and Environment for Statistical Computing. [http://www.R-project.org]

I I0. Farriol-Mathis N, Garavelli JS, Boeckmann B, Duvaud S, Gasteiger E, Gateau A, Veuthey AL, Bairoch A: Annotation of post-translational modifications in the Swiss-Prot knowledge base. Proteomics 2004, 4:1537-1550.

III. Tung CW, Ho SY: Computational identification of ubiquitylation sites from protein sequences. BMC Bioinformatics 2008, 9:31.

I 12. Mason SJ, Graham NE: Areas beneath the relative operating characteristics (ROC) and levels (ROL) curves: statistical significance and interpretation. Quart J R Meteorol Soc 2002, | 28:2| 45-2 |66

I 13. Yen HC, Elledge SJ: Identification of SCF ubiquitin ligase substrates by global protein stability profiling. Science 2008 , 322:923-929. 